\title{
Cytoprotection by a naturally occurring variant of ATP5G1 in Arctic ground squirrels
}

3

\section{Neel S. Singhal ${ }^{1^{*}}$, Meirong Bai ${ }^{2,3^{\star}}$, Evan M. Lee ${ }^{2,3}$, Shuo Luo ${ }^{2,3}$, Kayleigh R. Cook ${ }^{2,3}$, \&}

Dengke K. $\mathrm{Ma}^{2,3}$

${ }^{1}$ Department of Neurology, ${ }^{2}$ Cardiovascular Research Institute, and ${ }^{3}$ Department of Physiology, University of California-San Francisco, 555 South Mission Bay Blvd., San Francisco, CA, 94158, USA. *These authors contributed equally.

\section{Corresponding Author:}

Dengke K. Ma

Email: dengke.ma@ucsf.edu

Telephone: Office: 415-502-3386

Fax: 415-476-2283 
1 Abstract

2 Many organisms, from anaerobic bacteria to hibernating ground squirrels, have evolved

3 mechanisms to tolerate severe hypoxia or ischemia. In particular, the arctic ground squirrel

4 (AGS) has been shown to be highly resilient to ischemic and reperfusion injuries,

5 demonstrating an ability to withstand metabolic stress under hibernation conditions. Although

6 physiological adaptations are critical to ischemic tolerance in AGS, little is known about cellular

7 mechanisms underlying intrinsic AGS cell tolerance to metabolic stressors. Through cell

8 survival-based cDNA expression screens and comparative genomics, we have discovered that

9 in AGS, a cytoprotective variant of ATP5G1 helps confer improved mitochondrial metabolism

10 and cell resilience to metabolic stress. ATP5G1 encodes a proton-transporting subunit of the

11 mitochondrial ATP synthase complex. Ectopic expression in mouse cells and CRISPR/Cas9

12 base editing of the endogenous AGS locus revealed causal roles of one AGS-specific amino

13 acid substitution (leucine-32) in mediating the cytoprotective effects of AGS ATP5G1. We

14 provide evidence that AGS ATP5G1 promotes cell resilience to stress by modulating

15 mitochondrial morphological change and metabolic functions. Thus, our results identify a

16 naturally occurring variant of ATP5G1 from a mammalian hibernator that causally contributes

17 to intrinsic cytoprotection against metabolic stresses. 


\section{Introduction}

Arctic ground squirrels (AGS) survive harsh winter environmental conditions through hibernation. By virtue of their profound ability to suppress metabolism and core temperature, with body temperatures dropping below $0^{\circ} \mathrm{C}, \mathrm{AGS}$ are known as 'extreme' hibernators (1). Hibernation in AGS can last 7 months and is characterized by drastic $(>90 \%)$ reductions in basal metabolic rate, heart rate, and cerebral blood flow (2). Curiously, hibernation is interrupted periodically by interbout arousal (IBA) episodes in which temperature and cerebral ischemic and reperfusion injuries in numerous models, including brain and heart tissues after

11 cardiac arrest in vivo and hippocampal slice models derived from animals during an IBA (5-8).

12 This resilience to reperfusion injury does not depend on temperature of the animal or season.

13 In addition, AGS neural progenitor cells (NPCs) demonstrate resistance to oxygen and glucose

14 deprivation in vitro (9). Together, these studies suggest that in addition to physiological

15 adaptations, AGS possess cell autonomous genetic mechanisms that contribute to intrinsic tolerance to metabolic injury.

Proteomic and transcriptomic investigations have comprehensively catalogued the impact of season, torpor, and hibernation on cellular and metabolic pathways in several

19 different tissues of hibernating ground squirrels, including brain $(6,10-16)$. Although the

20 mechanisms underlying hibernating ground squirrel ischemia and hypothermia tolerance in the

21 brain are not fully elucidated, studies suggest that post-translational modifications, regulation of 22 cytoskeletal proteins, and upregulation of antioxidants play a prominent role (17-19). Recent 23 studies employing unbiased next-generation sequencing and bioinformatics approaches have 
1 adapting to hypothermia and hypoxia in ground squirrel and marmot species $(20,21)$. In neurons differentiated from 13-lined ground squirrel (13LGS) induced pluripotent stem cells,

3 Ou and colleagues found that hibernating ground squirrel microtubules retained stability upon exposure to hypothermia. The authors identified mitochondrial suppression of cold-induced reactive oxygen species (ROS) and preservation of lysosomal structure are key features of

6 ground squirrel cytoprotection, and that pharmacological inhibition of ROS production or lysosomal proteases recapitulates the hypothermia-tolerant phenotype in human cells (21). Taken together, these studies have provided important insights into pathways mediating

9 ischemia tolerance. However, these studies have not focused on specific proteins and their

10 cytoprotective properties uniquely evolved in hibernating ground squirrels. As such, we know

11 very little about mechanistic details underlying genetic contribution to intrinsic stress resilience

12 in ground squirrels. Using a functional genetic screening approach combined with analyses of

13 cell survival and mitochondrial phenotypes, we identified AGS transcripts imparting in vitro

14 cytoprotection to various metabolic stressors. We further use CRISPR/Cas9 base editing (22)

15 to determine functional importance of amino acid substitutions uniquely evolved in AGS, and identified AGS ATP5G $1^{\text {L32 }}$ as a mediator of key cytoprotective metabolic functions, suggesting

17 potential for targeting this component of ATP synthase for neuroprotective treatments.

Results

21 AGS neural cells exhibit marked resistance to metabolic stressors associated with

22 improvements in mitochondrial function and morphology 
1 NPCs (Figure $1 \mathrm{~A}$ and 1 Supplement). Although superficially indistinguishable, mouse and AGS NPCs demonstrate markedly different responses to metabolic stressors. When exposed

3 to hypoxia $\left(1 \% \mathrm{O}_{2}\right)$, hypothermia $\left(31^{\circ} \mathrm{C}\right)$, rotenone $(30 \mu \mathrm{M})$, or FCCP $(10 \mu \mathrm{M})$, AGS NPCs

4 exhibit profound resistance to cell death compared with mouse NPCs (Figure 1B), recapitulating resilient AGS phenotypes found in previous studies (5, 7-9). Moreover, measurement of in vitro oxygen consumption of AGS NPCs after sequential exposure to mitochondrial toxins demonstrates strikingly higher 'spare respiratory capacity' in response to FCCP (Figure 1C), indicating a greater metabolic reserve for stressors (23). Interestingly,

9 these functional improvements in mitochondrial function were also mirrored by changes in mitochondrial dynamic organization following exposure to FCCP. At baseline, mouse and AGS

11 cells had similar mitochondrial organization as evidenced by similar mean branch length and

12 number of cells with fragmented mitochondria (Figure 1D,E). Following FCCP, an uncoupling

13 agent that causes mitochondrial depolarization, mouse cells demonstrated large increases in

14 mitochondrial fission with concurrent decreases in mean branch length. By contrast, AGS cells

15 appeared largely resistant to mitochondrial fission induced by FCCP (Figure 1F,G). Together, these results demonstrate intrinsic differential cell survival and mitochondrial responses to

17 metabolic stresses between mouse and AGS NPCs.

\section{A cDNA library expression screen identifies AGS ATP5G1 as a cytoprotective factor}

To identify cytoprotective genes expressed in AGS, we constructed a normalized cDNA expression library from AGS NPCs and introduced the library to mouse NPCs by nucleofection.

22 Two days after AGS cDNA library nucleofection, we exposed cells to hypothermia $\left(31^{\circ} \mathrm{C}\right)$ for 3

23 days, hypoxia (1\%) for 2 days, or complex I inhibition (rotenone) for 3 days, respectively

24 (Figure 2A and 2 Supplement). We then isolated plasmids from surviving cells, amplified cDNA 
$1 \quad$ insert sequences by PCR and used next-generation sequencing to identify a total of 378

2 putative cytoprotective genes, three of which (Ags Atp5g1, Ags Manf, and Ags Calm1)

provided cytoprotection in all conditions (see Figure 2B and Supplemental Data File 1).

Since a portion of mouse NPCs survived metabolic stresses even without AGS cDNA

library expression, we anticipated false positive hits and focused on the nuclear-encoded

mitochondrial protein AGS ATP5G1, as it was one of three genes found to confer resilience

across all three metabolic stress paradigms (Figure 2B). We hypothesized that resistance to

mitochondrial insults may be related to uniquely evolved AGS amino acid substitutions in key

proteins. There are three substitutions and two insertions/deletions in the AGS ATP5G1 N-

terminal mitochondrial targeting sequence compared to the human and mouse sequences

11 (Figure 2C and 2 Supplement C). ATP5G1 is one of three ATP5G isoforms making up the C

12 subunit of ATP synthase, and is regulated distinctly from ATP5G2 of ATP5G3 (24-27). We

13 hypothesized a subset of AGS cDNA library screened cytoprotective variants contain uniquely

14 evolved amino acid substitutions. We identified uniquely evolved AGS proteins by comparing

15 sequence alignments of the screened cytoprotective candidates for 2 species of ground

squirrels (AGS and the 13LGS, Ictidomys tridecemlineatus) against 9 other reference species

across mammalian subclasses. Jensen Shannon Divergence (JSD) score, and average

ground squirrel-versus-other mammalian block substitution matrix (BLOSUM)-62 scores were

sequence conservation and difference from the background amino acid distribution. High JSD 
conservation analysis scored among the highest of all AGS-unique amino acid substitutions in screened cytoprotective proteins (28; Figure 2D and Supplemental Data File 2).

The N-terminal region of ATP5G proteins can undergo cleavage, but also modulate mitochondrial function directly, though the specifics of the mechanism are unknown (29). Although the three $\mathrm{C}$ subunit proteins are identical in sequence, they cannot functionally substitute for one another and are all required to constitute a fully functional C subunit $(29,30)$. To determine the relative levels of ATP5G1, -2, and -3 in mouse and AGS NPCs, we performed qRT-PCR analysis with species and transcript-specific primers. We found that in both mouse and AGS NPCs, expression of Atp5g3 or Atp5g2 is greater than that of Atp5g1, consistent with prior reports in human and mouse tissues $(25,29)$. However, the relative abundance of the Atp5g1 isoform is elevated nearly two-fold in AGS NPCs (Figure 2 Supplement D). Interestingly, the relative abundance of the mature ATP5G (subunit C) protein or complex $\mathrm{V}$ activity, is not different in mouse or AGS cells (Figure 2 Supplement E), suggesting that the beneficial effects of ATP5G1 could be mediated by interaction with other proteins.

Overexpression of the AGS variant of ATP5G1 in mouse NPCs confers cytoprotection in cells exposed to hypoxia, hypothermia, or rotenone. We found that this protective response is not present in NPCs overexpressing ATP5G1 ${ }^{\text {L32P }}$. Similarly, overexpression of the human ATP5G1 ${ }^{P 32 L}$, which mimics the wild-type AGS ATP5G1 variant, leads to enhanced cytoprotection in these conditions of metabolic stress compared to human ATP5G1 (Figure 2EG). The AGS ATP5G1 substitutions did not alter the mitochondrial localization of ATP5G1 (Figure 3 Supplement A-C), and importantly, recapitulated key features of the AGS resilient mitochondrial phenotype, including increasing spare respiratory capacity and reducing mitochondrial fission with reduced fragmentation and increased branch length of mitochondria 
1 (Figure 3A-D). Of note, the other identified ground squirrel amino acid substitutions (N34D,

T39P) did not demonstrate a loss of the protective effect on survival when overexpressed in

mouse NPCs exposed to hypoxia, hypothermia, or rotenone (Figure 3 Supplement D-F).

\section{Knock-in of AGS ATP5G1 ${ }^{\text {L32P }}$ alters the resilient phenotype of AGS cells}

Using the previously described adenine base editor (ABEmax; 22), we successfully

generated AGS cell lines homozygous for ATP5G $1^{\text {L32P }}$ by introducing a cytosine-to-thymine

substitution in the (-) strand of Ags Atp5g1 (Figure 4A,B). We isolated three clonal AGS NPC

lines harboring the desired knock-in mutation (ABE KI) and two clonal lines that underwent

editing and remained homozygous for the wild-type ATP5G1 gene (ABE WT). Compared to

11 ABEmax-treated AGS cells without successful knock-in (Figure 4 Supplement A), ABE KI cell

12 lines did not demonstrate differences in ATP5G mRNA, protein expression, or complex V

13 activity (Figure 4 Supplement B-E). In spite of stable ATP5G protein and complex V activity,

14 knock-in of the L32P residue resulted in reduced survival following exposure to hypoxia,

15 hypothermia, or rotenone (Figure 4C), again indicating that the cytoprotective effects of

ATP5G1 may be mediated by interactions with other proteins. In addition, we found the ABE KI

AGS NPCs exhibited marked reduction in 'spare respiratory capacity' and altered mitochondrial dynamics in response to FCCP treatment (Figure 4D-G). Taken together, these results support

a role for the AGS Leucine-32 substitution in the N-terminus of ATP5G1 in mediating

cytoprotection against metabolic stresses by modulating mitochondrial function.

\section{Discussion}

Previous studies have indicated that organisms capable of hibernation evolved a myriad 
1 the hibernation process $(20,21,31)$. However, we still know little about how specific protein-

2 coding genetic variants evolved in the AGS genome causally contribute to mechanisms

3 mediating intrinsic cytoprotective functions. We show that ex vivo cultured AGS NPCs

4 demonstrate remarkable intrinsic resilience to hypoxia, hypothermia, and other metabolic

5 stressors. Additionally, using an unbiased cDNA expression screening and bioinformatic

6 strategy, we identified numerous AGS transcripts and uniquely evolved AGS amino acid

7 substitutions potentially contributing to cytoprotection. We focused on discerning the protective effect of AGS ATP5G1, a nuclear-encoded mitochondrial protein, given that it was one of only

9 three genes identified in all three metabolic stress paradigms and the prominent mitochondrial

10 resilience phenotype of AGS NPCs. We hypothesize that analogous to amino acid

11 substitutions in several human proteins providing adaptive benefits (32-35), substitutions in

12 AGS ATP5G1 may underlie AGS adaptive mechanisms contributing to its robust cytoprotective

13 phenotype. Utilizing dCas9 ABE technology, we validated a unique Ags ATP5G $1^{L 32}$ amino acid

14 substitution in the mitochondrial targeting sequence of ATP5G1 that leads to improvements in

15 mitochondrial physiologic parameters. Thus, this study is among the first to our knowledge to use CRISPR base editing in a non-model organism, and demonstrates a potential role for

17 modulating ATP5G1 to enhance cytoprotection in ischemic diseases. Furthermore, the novel mitochondrial functional and morphological phenotypes established here can serve as a robust

19 in vitro paradigm to investigate the numerous other identified gene perturbations potentially

20 mediating the AGS resilient phenotype with implications for novel neuroprotective treatments

21 as well as promoting survival of neural stem cell grafts (36).

Mitochondrial metabolic dysfunction is central to ischemia and reperfusion injury.

23 Physiologic, transcriptomic, and proteomic studies have highlighted the importance of ketone 
1 post-translational protein modifications in the differential regulation of metabolic pathways in

2 hibernation $(10,39,40)$. We expanded this body of knowledge, by identifying altered mitochondrial dynamics and enhanced spare respiratory capacity in cells of hibernating species as potentially adaptive cellular mechanisms in hibernating animals. This mitochondrial phenotype is likely responsible for the broad resilience of AGS cells against a wide range of metabolic stressors. The downstream mediators of this substitution's beneficial effect remain to be determined, but do not appear to involve regulation of the mature ATP5G protein

abundance or complex $\mathrm{V}$ activity. represents a marker for cellular metabolic reserves (23) and is thought to be determined by the

11 oxidative phosphorylation machinery $(41,42)$. AGS demonstrate marked elevations in spare

12 respiratory capacity compared to mouse cells. While this is likely the result of AGS adaptations

13 in numerous proteins, the importance of the ATP5G1 variant is highlighted by our experimental

14 evidence demonstrating improvement in spare respiratory capacity in mouse NPCs over-

15 expressing AGS ATP5G1 variants and decreased spare respiratory capacity in AGS NPCs

with ATP5G1 L32P knock-in. A critical role for ATP5G1 in cellular energetics is also supported

17 by recent work uncovering ATP5G1 as one of the major effectors of the transcription factor,

BCL6, in regulating adipose tissue energetics as well as maintaining thermogenesis in response to hypothermia $(43,44)$.

Mitochondrial fission and fusion are regulated by cellular metabolic state and a host of

21 regulatory proteins, many of which have been implicated in cell survival response to stresses

22 (45). While metabolic stresses often lead to mitochondrial fission followed by apoptosis,

23 mitochondrial fusion and resistance to fission in response to stress are anti-apoptotic $(46,47)$.

24 Fusion is hypothesized to allow for complementation of damaged and dysfunctional 
1 mitochondria, and in states of metabolic stress, hyperfusion of mitochondria helps maintain

2 mitochondrial membrane potential and cell viability (48). The increase in fusion and

3 improvement in cell survival in mouse NPCs over-expressing AGS ATP5G1 and loss of

4 resilient metabolic phenotypes in AGS cells carrying ATP5G $1^{L 32 P}$ underscore the importance of

5 this protein in altering mitochondrial morphologic response to stresses and increasing the

6 metabolic oxidative capacity of cells.

contain a unique mitochondrial targeting sequence (MTS) providing a high degree of specificity

9 in regulating mitochondrial import and sorting. These mitochondrial targeting and processing

10 functions are regulated by the highly conserved mitochondrial membrane translocating protein

11 complexes (TOM and TIM) and MTS cleaving proteins, mitochondrial processing peptide

12 (MPP) and mitochondrial intermediate peptide (MIP). We did not find evidence that the

13 ATP5G1 MTS sequence differences between AGS and mouse or ABE WT and KI affected the

14 mitochondrial localization or expression levels of mature ATP5G protein, suggesting that the

15 phenotypic differences observed are not related to mitochondrial protein import or processing.

16 Furthermore, the putative ATP5G1 ten amino acid sequential MPP/MIP cleavage site

$17(\mathrm{xRx} \downarrow(\mathrm{F} / \mathrm{L} / \mathrm{I}) \mathrm{xx}(\mathrm{S} / \mathrm{T} / \mathrm{G}) \mathrm{xxxx} \downarrow ; 49)$ is also completely conserved in human, mouse, and AGS (see

18 Figure 2 Supplement C). In addition, the MTS of proteins can vary greatly in their length, and

19 many have speculated that in addition to organelle targeting the MTS also play important

20 regulatory roles (50). The cleaved ATP5G1 N-terminal mitochondrial targeting sequence has

21 been described to modulate mitochondrial function distinct from the functionally active C-

22 terminal protein (29). However, additional experiments will be required to understand how the

23 AGS substitutions in ATP5G1 mediate the cytoprotective effect. Interestingly, although

24 increased abundance of the Atp $5 g 1$ transcript in AGS neural cells may also contribute to the 
1 altered mitochondrial function in brown adipose tissue (27), the ABE KI cells did not

demonstrate a difference in ATP5G1 transcript abundance suggesting that ATP5G1

transcriptional isoform abundance does not underlie ATP5G1-related cytoprotection.

Further unraveling of the mechanisms underlying AGS mitochondrial and cellular

resilience to metabolic injuries holds the hope of identifying novel cytoprotective strategies that

will lead to improved cellular engineering strategies and treatments for ischemic diseases.

Systematic experimental investigation of the additional amino acid substitutions identified in

AGS will provide important insights into the pathways underlying intrinsic ischemic tolerance.

9 The use of CRISPR gene editing technologies coupled with detailed phenotypic analysis is a

10 unique and powerful approach to evaluate causal roles of genetic variants in conferring

11 phenotypic traits. Identification of such causal variants for stress resilience in AGS may help

12 develop pharmacological, gene therapy, or CRISPR/genome editing-based therapeutic

13 strategies to treat human ischemic disorders, including stroke and heart attack.

\section{Materials and Methods}

16 Cell culture. AGS NPCs (Neuronascent, Gaithersburg, MD, USA) and mouse NPCs (gift of

17 Song lab, Baltimore, MD) have been previously described $(9,51)$. They were grown under

18 standard conditions at $37^{\circ} \mathrm{C}$ and $5 \% \mathrm{CO}_{2}$ with NeuroCult basal media (STEMCELL, Vancouver,

19 BC, CA) with EGF (50 ng/ml, PeproTech, Inc., Rocky Hill, NJ, USA), FGF (100 ng/ml,

20 PeproTech, Inc.), heparin (0.002\%), and proliferation supplements (STEMCELL). Early

21 passage cultures (P2) were expanded and frozen and thawed in batches for use in

22 experiments. These cultures contain cells ubiquitously expressing the NPC marker, Nestin,

23 and the proliferation marker, Ki-67 (Figure S1). For in vitro modeling of metabolic stress, cells 
1 saturated with Nitrogen $/ 5 \% \mathrm{CO}_{2}$; ii) hypothermia in standard incubators maintained at lower

2 temperatures; and iii) complex I inhibition with the addition of rotenone to cell media.

4 DNA constructs and lentiviral transfection. The pHAGE-ATP5G plasmids were generated by

5 direct PCR and PCR fusions; and the point mutation plasmids generated using Q5 site-directed

6 mutagenesis (New England Biolabs, Beverly, MA, USA). For lentiviral transfection, the

7 plasmids with packaging plasmids were co-transfected into HEK293FT (with a ratio of

2:1.5:1.5) using Turbofect reagent (Thermo Fisher Scientific Inc., Waltham, MA, USA)

according to the manufacturer's instructions. Lentivirus-containing medium was filtered from

the post-transfection supernatant and used for transduction of HEK293T cells or mouse NPCs.

11 All lentivirus-infected cells were cultured in the medium containing Polybrene $(4 \mu \mathrm{g} / \mathrm{ml}$; Sigma

Generation of CRISPR Base-edited AGS cells. ATP5G $1^{\text {L32P }}$ NPCs were generated using the

dCas9 base editor, ABEmax (Addgene \#112095), as previously described. Briefly, a synthetic

sgRNA (TCCTCTAGTCTATTCAGGAA) was selected by manual inspection of the Ags Atp5g1

sequence for a PAM (NGG) site near the desired edit on the (-) strand of the gene. AGS NPCs

were nucleofected (Amaxa 4D, program DS113) in P3 solution (Lonza, Alpharetta, GA, USA)

containing pCMV ABEmax (500 ng/200,000 cells). Following a 48 hour recovery period, the

same cells were nucleofected with the synthetic sgRNA sequence above (100 pmol, Synthego,

Menlo Park, CA, USA). Cells were expanded and then clonally plated. Clones were screened

by PCR as the desired base edit also introduced a new Bfal restriction enzyme cutting site. 
1 utilized. Potential off-target effects of CRISPR/Cas9 cleavage were analyzed by Sanger

2 sequencing of the top 5 predicted off-target genomic locations [https://mit.crispr.edu], which

3 demonstrated a lack of indels for all clones used in subsequent analysis.

Cell death assay. Mouse and AGS cells were plated in 24 or 96 -well plates and grown to $70 \%$ confluence. Cells were exposed to metabolic stress paradigms as above, and detached and floating cells collected by centrifugation and washed with $1 \mathrm{ml}$ PBS. The collected cells were resuspended with $200 \mu \mathrm{l}$ PBS with addition of $0.2 \mu$ Sytox blue $(1 \mu \mathrm{M}$; Thermo Fisher Scientific) or propidium iodide (concentration) for an additional $5 \mathrm{~min}$. The fluorescence intensity was measured for individual cells using automated cytometry (Arthur, Nanoentek, Waltham, MA,

11 USA) or flow cytometry (BD Biosciences, San Jose, CA, USA) within 20 min of staining, and

12 the percentage of cell death quantified using the FlowJo software.

cDNA Library generation, screening, and identification of AGS amino acid substitutions. RNA

was isolated from AGS NSC/NPC cells grown under standard conditions. A normalized cDNA

17 from RNA extracted from AGS NPCs. Library quality and normalization is shown in Figure 2

18 Supplement A and B). For library screening, plates containing $1 \times 10^{7}$ mouse NPCs cells were grown in triplicate and nucleofected with 200,000 clones each. Plates were exposed to one of

20 three metabolic stress conditions (hypoxia, hypothermia, or rotenone treatment) for 48-96

21 hours. Following this treatment, plasmid DNA was purified from surviving cells and PCRamplified AGS cDNA inserts subjected to next-generation sequencing. Resulting fastq files

23 were trimmed (Trim Galore!) and mapped to the Ictidomys Tridecemlineatus genome 
1 amino acid substitutions. Briefly, protein sequences of mapped genes were queried by gene symbol and downloaded from OrthoDBv10 (52) for 10 species (13LGS, Mus musculus, Rattus norvegicus, Sorex araneus, Pongo abelii, Homo sapiens, Equus caballus, Bos taurus,

4 Oryctolagus cuniculus, Sus scrofa). OrthoDB data was filtered by matching records against accepted GeneCards aliases for each gene. Multiple records per species were resolved using maximum percent identity against the accepted human, mouse, and 13LGS sequences, such

that only one record per species was used for alignment. AGS protein sequences were downloaded from the Entrez Protein database. Multiple AGS isoforms were resolved by best identity match to the OrthoDB sequence data. The final protein sequence set was aligned with KAlign 2.04 (53). From aligned sequences, GS-specific residue substitutions were defined as

11 amino acid variants present in 13LGS and AGS sequences and present in no other included

12 species. For each GS-specific residue, sequence weights, JSD, and average GS-versusoutgroup BLOSUM62 scores were calculated as described previously (28). BLOSUM62 scores were used instead of point-accepted mutation scores in order to prioritize protein sequence changes with higher probability of potential chemical and functional difference. JSD was used to capture sequence conservation and difference from the background amino acid distribution. BLOSUM62 scores were calculated for GS residues against all other mammalian species sequences and averaged to give GS vs Outgroup BLOSUM62. For the entire screened cytoprotective protein dataset, JSD and BLOSUM62 score were plotted for individual genes of

20 interest against the remaining dataset. 
1 to the manufacturer's instructions. Basal respiration and ATP production were calculated to

2 evaluate mitochondrial respiratory function according to the manufacturer's instructions. After

3 the measurement, cells were harvested to count the cell number, and each plotted value was

4 normalized relative to the number of cells used. Briefly, NPCs were seeded (25,000 cells/well)

5 into each well of $\mathrm{XF}^{\mathrm{e}} 96$ cell culture plates and were maintained in standard culture media. After

$6 \quad 2-3$ days in culture, cells were equilibrated in unbuffered $\mathrm{XF}^{\mathrm{e}}$ assay medium (Seahorse

7 Bioscience) supplemented with glucose (4.5 g/L), sodium pyruvate $(25 \mathrm{mg} / \mathrm{L})$ and transferred

8 to a non- $\mathrm{CO}_{2}$ incubator for $1 \mathrm{~h}$ before measurement. Oxygen consumption rate (OCR) was

9 measured with sequential injections of $1-2 \mu \mathrm{M}$ oligomycin, 2-4 $\mu \mathrm{M}$ FCCP and each $0.5 \mu \mathrm{M}$ of

10 rotenone/antimycin $\mathrm{A}$.

12 Analysis of mitochondrial respiratory chain complex activity. Analysis of mitochondrial

13 respiratory chain complex V activity was measured with Complex V Mitocheck kit (Cayman

14 Chemical, Ann Arbor, MI, USA). Mitochondrial extracts $(50 \mu \mathrm{g})$ were obtained as previously

15 described (54) and used to measure time-dependent absorbance alterations on a multi-well

16 plate reader (SprectraMax, Molecular Devices, San Jose, CA, USA).

Mitochondrial Dynamic Morphology Assessment. Mitochondrial morphology and fission/fusion

19 is assessed in mouse and AGS NPCs nucleofected with mCherry-Cox8 as a mitochondrial

20 marker and grown on glass coverslips in standard media. Cells are allowed to recover for 48

21 hours and then fixed with paraformaldehyde (4\%) one hour following treatment with FCCP (1

$22 \mathrm{uM}$ ) or DMSO. High magnification images of cells are captured by confocal microscopy and

23 mitochondrial morphological characteristics were assessed with the Mitochondrial Network 
Analysis (MiNA) toolset in J-image as previously described $(55,56)$. Briefly, the plugin converts

confocal images to binary pixel features and analyzes the spatial relationship between pixels.

The parameters analyzed are: (i) individual mitochondrial structures; ii) networked

mitochondrial; and iii) the average of length of rods/branches. Twenty randomly chosen fields

containing 30-50 cells were used to quantify the morphological pattern and network branch

lengths of mitochondria. We classify the mitochondrial morphology as fragmented when the

appearance is completely dotted with branch lengths $<1.8 \mu \mathrm{m}$.

Immunoblot analysis. Laemmli loading buffer (Bio-Rad Lab, Hercules, CA, USA) plus 5\% $\beta$ -

mercaptoethanol was added to protein extracts from cell pellets before heating at $95^{\circ} \mathrm{C}$ for

$115 \mathrm{~min}$. Around $30 \mu \mathrm{g}$ of whole cell protein lysate samples were separated on mini-PROTEIN

12 GTX precast gels, and transferred to nitrocellulose membranes (Bio-rad). After blocking in TBS

with $5 \%$ milk at $4{ }^{\circ} \mathrm{C}$, followed by incubation with secondary antibodies at room temperature for

1h. Immunoreactivity was visualized by the ECL chemiluminescence system (Bio-rad) on

standard film. The antibodies were ATP Synthase C subunit (ab-181243, 1:1000, Abcam,

Cambridge, MA), HSP90 (sc-69703, 1:1000, Santa Cruz Biotechnology, Dallas, TX, USA).

Immunofluorescence and confocal microscopy. For immunocytochemistry of mammalian cells,

21 AGS and mouse NSC/NPC cells were seeded on laminin-coated coverslips (Neuvitro,

22 Vancouver, WA, USA) within 24-well plates. The cells were fixed with $4 \%$ paraformaldehyde in

23 PBS, washed with PBS, and permeabilized with $0.02 \%$ Triton X-100 in PBS for 10 min. 
1 Nestin (MAB2736, 1:50, R\&D Systems, Cambridge, MA, USA) or Ki-67 (NB600-1252, 1:500,

2 Novus Biologicals, Littleton, CO, USA) in blocking buffer overnight at $4^{\circ} \mathrm{C}$. The Nestin antibody

3 was detected using goat anti-mouse AlexaFluor 488 or 647 (1:1000; Jackson

4 ImmunoResearch Laboratories Inc., West Grove, PA, USA) and the Ki-67 antibody was

detected using AlexaFluor 488 goat anti-rabbit (1:1000; Jackson Immunoresearch) or Cy3-

conjuated donkey anti-rabbit (1:500; EMD Millipore, Burlington, MA, USA) in blocking buffer.

Cells were washed with PBS after primary and secondary antibody staining. Stained cells were

overlaid with Fluoroshield mounting medium with DAPI (Abcam) to label nucleus. Fluorescence

microscopy was performed with a Leica confocal microscope using the following fluorescence

filters: DAPI (405 nm excitation); Су3 (551 nm excitation); AlexaFluor 647 (651 nm excitation);

11 and GFP/AlexaFluor 488 ( $488 \mathrm{~nm}$ excitation). For comparison across conditions, identical light-

12 exposure levels were used.

Quantitative RT-PCR. RNA was extracted from approximately 200,000 mouse or AGS NPCs

per condition according to manufacturer instructions (Quick-RNA MiniPrep kit; Irvine, CA,

USA). Total RNA was reverse transcribed into cDNA (Bimake, Houston, TX, USA), and real-

17 time PCR was performed (LightCycler96, Roche, Basel, CHE) with SYBR Green (Thermo

Fisher Scientific) as a dsDNA-specific binding dye. Quantitative RT-PCR conditions were $95^{\circ} \mathrm{C}$

19 for denaturation, followed by 45 cycles of: $10 \mathrm{~s}$ at $95^{\circ} \mathrm{C}, 10 \mathrm{~s}$ at $60^{\circ} \mathrm{C}$, and $20 \mathrm{~s}$ at $72^{\circ} \mathrm{C}$.

20 Species-specific primers for each transcript were used (for list see Supplemental Table 1).

21 Melting curve analysis was performed after the final cycle to examine the specificity of primers

22 in each reaction. Relative abundance of each Atp5g isoform as a fraction of total Atp5g was

23 calculated by $\Delta \Delta \mathrm{CT}$ method and normalized to $\mathrm{Rp} / 27$. 
1 Statistical Analysis. Data were analyzed using GraphPad Prism Software (Graphpad, San

2 Diego, CA) and presented as means \pm S.E. unless otherwise specified, with $P$-values

3 calculated by two-tailed unpaired Student's t-tests or two-way ANOVA (comparisons across

4 more than two groups) adjusted with the Bonferroni's correction. No randomization or blinding

5 was used and no power calculations were done to detect a pre-specified effect size.

7 Data availability. The data that support the findings of this study are available from the corresponding author upon reasonable request.

\section{Acknowledgments}

11 NSS and MB receive support from the American Heart Association, 18CDA34030443 and

12 19POST34381071, respectively. DKM receives support from National Institutes of Health grant

13 R01GM117461, Pew Scholar Award, Curci Faculty Scholar Award from the Innovative

14 Genomics Institute, and Packard Fellowship in Science and Engineering. The authors

15 acknowledge use of sponsored core facilities including the UCSF Laboratory for Cell Analysis 16 (P30CA082103).

\section{Author Contributions}

19 NSS, MB, ELM, DKM conceived of the work, designed the experiments, and interpreted the

20 data. NSS, MB, ELM, SL, and KRC performed experiments and acquired and analyzed the

21 data. NSS drafted and along with all other authors revised the manuscript. DKM

22 conceptualized and supervised the project. 


\section{Figure Legends}

Figure 1: Phenotypic characteristics of Mouse and AGS NPCs. (A.) Confocal image of Mouse (top) and AGS (bottom) NPCs demonstrating similar morphology and expression of Nestin (red) and Ki-67 (teal) in nearly all cultured cells of both species. (B.) AGS NPCs exhibit increased cell survival when exposed to hypoxia $(1 \%, 24 \mathrm{hrs})$, hypothermia $\left(31^{\circ} \mathrm{C}, 24 \mathrm{hrs}\right)$, or rotenone (10 $\mu \mathrm{M}, 16 \mathrm{hrs})$. Bar graphs represent the mean of 3 independent experiments with 3 replicates/condition. (C.) Seahorse XF analyzer assay of cultured mouse and AGS NPCs sequentially exposed to i) oligomycin $(1 \mu \mathrm{M})$, ii) FCCP $(2 \mu \mathrm{M})$, and iii) rotenone $(0.5 \mu \mathrm{M})$ showing enhanced FCCP-stimulated oxygen consumption (spare respiratory capacity). Data represents the mean of 3 independent experiments with 4-6 replicates/species. (D and F.) Representative confocal images of mouse (left) and AGS (right) NPCs expressing the mitochondrial marker mCherry-mito7 to demonstrate mitochondrial morphology at baseline (D.) and one hour following treatment with FCCP (F.). Scale bar represents $10 \mu \mathrm{m}$. (E and G.) Percent of mitochondria with fragmented morphology (left panel) and the mean branch length (right panel) of mitochondrial networks of NPCs expressing mCherry-mito7. Data obtained from 30 cells/species/condition. ${ }^{*} P<0.05 ;{ }^{* * *} P<0.001$.

\section{Figure 2: AGS cDNA library survival screen identifies AGS ATP5G1 as a cytoprotective} factor. (A.) AGS NPC cDNA was introduced into mouse NPCs by nucleofection. Cells were screened for survival after exposure to hypoxia $(1 \%, 48 \mathrm{hrs})$, hypothermia $\left(31^{\circ} \mathrm{C}, 72 \mathrm{hrs}\right)$, or rotenone (20 $\mu \mathrm{M}, 48 \mathrm{hrs})$ to identify AGS cytoprotective factors. (B.) Venn-diagram demonstrating the number of cytoprotective proteins identified by next-generation sequencing of plasmids isolated from cells surviving each condition of the cDNA library screen. (C.) Truncated sequence alignments demonstrating key GS AA substitutions (blue highlight) for 
1 ATP5G1, one of the three proteins imparting survival in all three screens. (D.) Ground squirrel-

2 unique amino acid substitutions are plotted as a function of BLOSUM62 score and Jensen-

3 Shannon Divergence (JSD) score. Ground squirrel unique AA substitutions with a higher

4 probability of functional consequence are in the denoted red quadrant (high JSD values and

5 low BLOSUM62 scores). The red dot represents the ATP5G $1^{\text {L32P }}$ substitution; orange dots

6 represent the other ATP5G1 substitutions. (E-G.) Mouse NPCs expressing human ATP5G1, AGS ATP5G1, AGS ATP5G $1^{\mathrm{L} 32 \mathrm{P}}$, human ATP5G1 ${ }^{\mathrm{P} 32 \mathrm{~L}}$, or empty vector (EV) and exposed to 24 hours of $1 \% \mathrm{O}_{2}(\mathrm{E}),. 31^{\circ} \mathrm{C}(\mathrm{F}$.$) , or 20 \mathrm{mM}$ rotenone (G.). Cell death was determined by flow cytometry for propidium iodide and experiments are mean of 3 independent experiments with 3 replicates/genotype/condition, ${ }^{\star} P<0.05$ or ${ }^{* *} P<0.01$ vs EV; $\delta<0.05$ vs human ATP5G1.

Figure 3: Overexpression of AGS ATP5G1 in mouse NPCs recapitulates AGS metabolic phenotypes, which is dependent on the uniquely evolved leucine-32. (A.) Seahorse XF analyzer assay of cultured mouse NPCs expressing human ATP5G1, AGS ATP5G1, AGS ATP5G1 ${ }^{\mathrm{L} 32 \mathrm{P}}$, human ATP5G1 ${ }^{\mathrm{P} 32 \mathrm{~L}}$, or empty vector and sequentially exposed to i) oligomycin $(\mu \mathrm{M})$, ii) FCCP $(2 \mu \mathrm{M})$, and iii) rotenone $(0.5 \mu \mathrm{M})$ showing increased FCCP-stimulated oxygen consumption (spare respiratory capacity) with AGS ATP5G1. Substitution of the AGS leucine32 results in reduced spare respiratory capacity.

(B-D.) Representative confocal images (D.)

19 of mitochondrial networks in mouse NPCs expressing human, AGS, and mutant forms of 20 mCherry-ATP5G1 one hour following treatment with FCCP. Percent of mitochondria with 21 fragmented morphology (B.) and the mean branch length (C.) of mitochondrial networks of 22 ATP5G1 overexpressing mouse NPCs. Data obtained from 30 cells/condition. ${ }^{*} P<0.05$; $23{ }^{\star \star} P<0.01$ vs. human ATP5G1; $\delta<0.05$ vs AGS ATP5G1. Scale bar in (D.) represents 5 um. 
1 Figure 4: CRISPR base editing to generate ATP5G1 ${ }^{\text {L32P }}$ AGS NPCs results in a partial

2 loss of AGS metabolic resilient phenotypes. (A.) AGS ATP5G1 CRISPR base editing

3 strategy. To create AGS cells with the human amino acid substitution at leucine-32, AGS cells

4 transiently expressing ABEmax were nucleofected with an sgRNA (blue underline) directed

5 towards a PAM site (green underline) on the (-) strand to target conversion of adenine to

6 guanine, which on the $(+)$ strand is a cytosine to thymine $\left(^{*}\right)$. (B.) Sequencing data from a

7 successfully edited clonal AGS cell line demonstrating the cytosine to thymine base edit

8 resulting in the desired leucine to proline knock-in cell line. (C.) AGS ATP5G1 ${ }^{\text {L32P }}$ (ABE KI)

9 NPCs exhibit decreased cell survival compared to unedited AGS cells (ABE WT) when

10 exposed to hypoxia $(1 \%, 24 \mathrm{hrs})$, hypothermia $\left(31^{\circ} \mathrm{C}, 72 \mathrm{hrs}\right)$, or rotenone $(10 \mu \mathrm{M}, 16 \mathrm{hrs})$. Bar

11 graphs represent the mean of 3 independent experiments with 3 replicates/condition. (D.)

12 Seahorse XF analyzer assay of cultured ABE KI and WT cells sequentially exposed to i)

13 oligomycin (1uM), ii) FCCP (2uM), and iii) rotenone (0.5uM) showing enhanced FCCP-

14 stimulated oxygen consumption (spare respiratory capacity). Data represents the mean of 3

15 independent experiments with 4-6 replicates/species. (E.) Representative confocal images of

$16 \mathrm{ABE}$ WT (left) and ABE KI (right) NPCs expressing the mitochondrial marker mCherry-mito7 to

17 demonstrate mitochondrial morphology one hour following treatment with FCCP. Scale bar

18 represents 5 um. (F and G.) Percent of mitochondria with fragmented morphology (F.) and the

19 mean branch length $(\mathrm{G})$ of mitochondrial networks of ABE WT and KI NPCs expressing

20 mCherry-mito7. Data obtained from 50-60 cells/genotype. ${ }^{\star} P<0.05 ;{ }^{* \star} P<0.01$. 
1 Figure 1 Supplement: Mouse and AGS NPCs express Nestin and Ki-67. (A-B.)

2 Representative live brightfield microscopy image of mouse and AGS NPCs. (C-D.)

3 Representative fluorescent microscopy images of fixed mouse and AGS NPCs immunolabeled

4 with Nestin (green) and Ki-67 (red). (E.) Quantitative assessment of mouse and AGS NPCs

5 demonstrates that nearly all cells express both Nestin and Ki-67. Cell counts from 25

6 microscopic fields/cell line.

Figure 2 Supplement: cDNA library construction and ATP5G expression in mouse and

AGS NPCs. (A.) Agarose gel of 11 randomly chosen AGS cDNA library clones prior to

11 normalized AGS cDNA library. (C.) ATP5G1 sequence alignment in human, mouse, AGS, and

12 rat demonstrating variability in the mitochondrial targeting sequence (alignment visualized

13 using PRALINE, http://www.ibi.vu.nl/programs/pralinewww/). The * indicates putative

14 mitochondrial processing peptide (MPP) and mitochondrial intermediate peptide (MIP)

15 cleavage sites. (D.) qRT-PCR for ATP5G1, ATP5G2, and ATP5G3 demonstrating increased

16 relative abundance of ATP5G1 in AGS NPCs. Data from 4 independent experiments

17 performed in triplicate. ${ }^{*} P<0.05^{\star * *} P<0.001$. (E.) Representative western blot images

18 demonstrating the relative abundance of ATP5G protein isoforms is similar in mouse and AGS.

19 (F.) Quantification of western blots from 4 independent experiments with 2-3 replicates each.

20 (G.) Complex V activity measured in mitochondrial extracts from mouse and AGS. Data from 3

21 independent experiments with 3 replicates each.

23 Figure 3 Supplement: AGS ATP5G1 is targeted to mitochondria and overexpression of 24 ATP5G1 $^{\mathrm{D} 34 \mathrm{~N}}$ and ATP5G1 ${ }^{\mathrm{T39P}}$ mutant constructs do not alter cytoprotection. (A.) Confocal 
1 images of mouse NPCs expressing mCherry-AGS ATP5G1 and GFP-Cox8 demonstrating co-

2 localization of the two proteins. (B-D.) Mouse NPCs expressing mutant AGS ATP5G1

3 isoforms (ATP5G1 ${ }^{\mathrm{D} 34 \mathrm{~N}}$, ATP5G $1^{\mathrm{T} 39 \mathrm{P}}$ ) or empty vector $(\mathrm{EV})$ and exposed to 24 hours of $1 \% \mathrm{O}_{2}$

4 (B.), $31^{\circ} \mathrm{C}$ (C.), or $20 \mathrm{mM}$ rotenone (D.). Bar graphs represent data from 3 independent 5 experiments with 3 replicates/genotype/condition, ${ }^{*} P<0.05$ vs EV.

7 Figure 4 Supplement: ATP5G expression in ABE cell lines. (A.) Sequencing data from an unsuccessfully edited clonal AGS cell line demonstrating the preservation of the wild-type

9 sequence ( ${ }^{*}$ indicates wild-type thymidine). (B.) qRT-PCR for ATP5G1, ATP5G2, and ATP5G3

10 demonstrating the same relative abundance of ATP5G1 in ABE WT and ABE KI NPCs. Data

11 from 3 independent experiments performed in triplicate. (C.) Representative western blot

12 images demonstrating the relative abundance of ATP5G protein isoforms is similar in ABE WT

13 and ABE KI cells. (D.) Quantification of western blots from 4 independent experiments with 2-3

14 replicates each. (E.) Complex V activity measured in mitochondrial extracts from ABE WT and

15 ABE KI AGS NPCs. Data from 3 independent experiments with 3 replicates each. 


\section{References}

1. Barnes BM. Freeze avoidance in a mammal: body temperatures below 0 degree $\mathrm{C}$ in an Arctic hibernator. Science. 1989;244(4912):1593-5.

2. Buck CL, Barnes BM. Effects of ambient temperature on metabolic rate, respiratory quotient, and torpor in an arctic hibernator. Am J Physiol Regul Integr Comp Physiol. 2000;279(1):R255-62.

3. Drew KL, Harris MB, LaManna JC, Smith MA, Zhu XW, Ma YL. Hypoxia tolerance in mammalian heterotherms. J Exp Biol. 2004;207(Pt 18):3155-62.

4. Karpovich SA, Toien O, Buck CL, Barnes BM. Energetics of arousal episodes in hibernating arctic ground squirrels. J Comp Physiol B. 2009;179(6):691-700.

5. Dave KR, Anthony Defazio R, Raval AP, Dashkin O, Saul I, Iceman KE, et al. Protein kinase C epsilon activation delays neuronal depolarization during cardiac arrest in the euthermic arctic ground squirrel. J Neurochem. 2009;110(4):1170-9.

6. Quinones QJ, Zhang Z, Ma Q, Smith MP, Soderblom E, Moseley MA, et al. Proteomic Profiling Reveals Adaptive Responses to Surgical Myocardial Ischemia-Reperfusion in Hibernating Arctic Ground Squirrels Compared to Rats. Anesthesiology. 2016;124(6):1296-310.

7. Bhowmick S, Moore JT, Kirschner DL, Drew KL. Arctic ground squirrel hippocampus tolerates oxygen glucose deprivation independent of hibernation season even when not hibernating and after ATP depletion, acidosis, and glutamate efflux. J Neurochem. 2017;142(1):160-70.

8. Bogren LK, Olson JM, Carpluk J, Moore JM, Drew KL. Resistance to systemic inflammation and multi organ damage after global ischemia/reperfusion in the arctic ground squirrel. PLoS One. 2014;9(4):e94225.

9. Drew KL, Wells M, McGee R, Ross AP, Kelleher-Andersson J. Arctic ground squirrel neuronal progenitor cells resist oxygen and glucose deprivation-induced death. World J Biol Chem. 2016;7(1):168-77.

10. Ballinger MA, Hess C, Napolitano MW, Bjork JA, Andrews MT. Seasonal changes in brown adipose tissue mitochondria in a mammalian hibernator: from gene expression to function. Am $\mathrm{J}$ Physiol Regul Integr Comp Physiol. 2016;311(2):R325-36.

11. Chang $\mathrm{H}$, Jiang $\mathrm{S}$, Ma X, Peng $X$, Zhang J, Wang Z, et al. Proteomic analysis reveals the distinct energy and protein metabolism characteristics involved in myofiber type conversion and resistance of atrophy in the extensor digitorum longus muscle of hibernating Daurian ground squirrels. Comp Biochem Physiol Part D Genomics Proteomics. 2018;26:20-31.

12. Gehrke S, Rice S, Stefanoni D, Wilkerson RB, Nemkov T, Reisz JA, et al. Red Blood Cell Metabolic Responses to Torpor and Arousal in the Hibernator Arctic Ground Squirrel. J Proteome Res. 2019;18(4):1827-41.

13. Hampton M, Melvin RG, Andrews MT. Transcriptomic analysis of brown adipose tissue across the physiological extremes of natural hibernation. PLoS One. 2013;8(12):e85157.

14. Luan Y, Ou J, Kunze VP, Qiao F, Wang Y, Wei L, et al. Integrated transcriptomic and metabolomic analysis reveals adaptive changes of hibernating retinas. J Cell Physiol. 2018;233(2):1434-45.

15. Andrews MT. Molecular interactions underpinning the phenotype of hibernation in mammals. $J$ Exp Biol. 2019;222(Pt 2).

16. Hindle AG, Grabek KR, Epperson LE, Karimpour-Fard A, Martin SL. Metabolic changes associated with the long winter fast dominate the liver proteome in 13-lined ground squirrels. Physiol Genomics. 2014;46(10):348-61.

17. Bhowmick S, Drew KL. Arctic ground squirrel resist peroxynitrite-mediated cell death in response to oxygen glucose deprivation. Free Radic Biol Med. 2017;113:203-11.

18. Lee YJ, Miyake S, Wakita H, McMullen DC, Azuma Y, Auh S, et al. Protein SUMOylation is massively increased in hibernation torpor and is critical for the cytoprotection provided by ischemic preconditioning and hypothermia in SHSY5Y cells. J Cereb Blood Flow Metab. 2007;27(5):950-62. 
19. Tessier SN, Wu CW, Storey KB. Molecular control of protein synthesis, glucose metabolism, and apoptosis in the brain of hibernating thirteen-lined ground squirrels. Biochem Cell Biol. 2019;97(5):536-44.

20. Bai L, Liu B, Ji C, Zhao S, Liu S, Wang R, et al. Hypoxic and Cold Adaptation Insights from the Himalayan Marmot Genome. iScience. 2019;11:519-30.

21. Ou J, Ball JM, Luan Y, Zhao T, Miyagishima KJ, Xu Y, et al. iPSCs from a Hibernator Provide a Platform for Studying Cold Adaptation and Its Potential Medical Applications. Cell. 2018;173(4):851-63 e16.

22. Koblan LW, Doman JL, Wilson C, Levy JM, Tay T, Newby GA, et al. Improving cytidine and adenine base editors by expression optimization and ancestral reconstruction. Nat Biotechnol. 2018;36(9):843-6.

23. Nicholls DG, Budd SL. Mitochondria and neuronal survival. Physiol Rev. 2000;80(1):315-60. 24. De Grassi A, Lanave C, Saccone C. Evolution of ATP synthase subunit $c$ and cytochrome $c$ gene families in selected Metazoan classes. Gene. 2006;371(2):224-33.

25. Gay NJ, Walker JE. Two genes encoding the bovine mitochondrial ATP synthase proteolipid specify precursors with different import sequences and are expressed in a tissue-specific manner. EMBO J. 1985;4(13A):3519-24.

26. Wigington CP, Morris KJ, Newman LE, Corbett AH. The Polyadenosine RNA-binding Protein, Zinc Finger Cys3His Protein 14 (ZC3H14), Regulates the Pre-mRNA Processing of a Key ATP Synthase Subunit mRNA. J Biol Chem. 2016;291(43):22442-59.

27. Andersson U, Houstek J, Cannon B. ATP synthase subunit c expression: physiological regulation of the P1 and P2 genes. Biochem J. 1997;323 ( Pt 2):379-85.

28. Capra JA, Singh M. Predicting functionally important residues from sequence conservation. Bioinformatics. 2007;23(15):1875-82.

29. Vives-Bauza C, Magrane J, Andreu AL, Manfredi G. Novel role of ATPase subunit C targeting peptides beyond mitochondrial protein import. Mol Biol Cell. 2010;21(1):131-9.

30. Sangawa H, Himeda T, Shibata H, Higuti T. Gene expression of subunit c(P1), subunit $c(P 2)$, and oligomycin sensitivity-conferring protein may play a key role in biogenesis of H+-ATP synthase in various rat tissues. J Biol Chem. 1997;272(9):6034-7.

31. Ballinger MA, Schwartz C, Andrews MT. Enhanced oxidative capacity of ground squirrel brain mitochondria during hibernation. Am J Physiol Regul Integr Comp Physiol. 2017;312(3):R301-R10. 32. Simonson TS, Yang Y, Huff CD, Yun H, Qin G, Witherspoon DJ, et al. Genetic evidence for high-altitude adaptation in Tibet. Science. 2010;329(5987):72-5.

33. Song D, Li LS, Arsenault PR, Tan Q, Bigham AW, Heaton-Johnson KJ, et al. Defective Tibetan PHD2 binding to p23 links high altitude adaption to altered oxygen sensing. J Biol Chem.

2014;289(21):14656-65.

34. Xiang K, Ouzhuluobu, Peng Y, Yang Z, Zhang X, Cui C, et al. Identification of a Tibetan-specific mutation in the hypoxic gene EGLN1 and its contribution to high-altitude adaptation. Mol Biol Evol. 2013;30(8):1889-98.

40 35. Yates CM, Sternberg MJ. The effects of non-synonymous single nucleotide polymorphisms 41 (nsSNPs) on protein-protein interactions. J Mol Biol. 2013;425(21):3949-63.

36. Bernstock JD, Peruzzotti-Jametti L, Ye D, Gessler FA, Maric D, Vicario N, et al. Neural stem cell transplantation in ischemic stroke: A role for preconditioning and cellular engineering. J Cereb Blood Flow Metab. 2017;37(7):2314-9.

37. Brown JC, Staples JF. Substrate-specific changes in mitochondrial respiration in skeletal and cardiac muscle of hibernating thirteen-lined ground squirrels. J Comp Physiol B. 2014;184(3):401-14. 38. Xu Y, Shao C, Fedorov VB, Goropashnaya AV, Barnes BM, Yan J. Molecular signatures of mammalian hibernation: comparisons with alternative phenotypes. BMC Genomics. 2013;14:567. 39. Chung DJ, Szyszka B, Brown JC, Huner NP, Staples JF. Changes in the mitochondrial phosphoproteome during mammalian hibernation. Physiol Genomics. 2013;45(10):389-99. 
40. Herinckx G, Hussain N, Opperdoes FR, Storey KB, Rider MH, Vertommen D. Changes in the phosphoproteome of brown adipose tissue during hibernation in the ground squirrel, Ictidomys tridecemlineatus. Physiol Genomics. 2017;49(9):462-72.

41. Pfleger J, He M, Abdellatif M. Mitochondrial complex II is a source of the reserve respiratory capacity that is regulated by metabolic sensors and promotes cell survival. Cell Death Dis. 2015;6:e1835.

42. Yadava N, Nicholls DG. Spare respiratory capacity rather than oxidative stress regulates glutamate excitotoxicity after partial respiratory inhibition of mitochondrial complex I with rotenone. J Neurosci. 2007;27(27):7310-7.

43. Kutyavin $\mathrm{VI}$, Chawla $\mathrm{A}$. BCL6 regulates brown adipocyte dormancy to maintain thermogenic reserve and fitness. Proc Natl Acad Sci U S A. 2019;116(34):17071-80.

44. Senagolage MD, Sommars MA, Ramachandran K, Futtner CR, Omura Y, Allred AL, et al. Loss of Transcriptional Repression by BCL6 Confers Insulin Sensitivity in the Setting of Obesity. Cell Rep. 2018;25(12):3283-98 e6.

45. Labbe K, Murley A, Nunnari J. Determinants and functions of mitochondrial behavior. Annu Rev Cell Dev Biol. 2014;30:357-91.

46. Abdelwahid E, Yokokura T, Krieser RJ, Balasundaram S, Fowle WH, White K. Mitochondrial disruption in Drosophila apoptosis. Dev Cell. 2007;12(5):793-806.

47. Chen H, McCaffery JM, Chan DC. Mitochondrial fusion protects against neurodegeneration in the cerebellum. Cell. 2007;130(3):548-62.

48. Gomes LC, Di Benedetto G, Scorrano L. During autophagy mitochondria elongate, are spared from degradation and sustain cell viability. Nat Cell Biol. 2011;13(5):589-98.

49. Gakh O, Cavadini P, Isaya G. Mitochondrial processing peptidases. Biochimica et Biophysica Acta (BBA) - Molecular Cell Research. 2002;1592(1):63-77.

50. Harbauer AB, Zahedi RP, Sickmann A, Pfanner N, Meisinger C. The protein import machinery of mitochondria-a regulatory hub in metabolism, stress, and disease. Cell Metab. 2014;19(3):357-72.

51. Ma DK, Ponnusamy K, Song MR, Ming GL, Song H. Molecular genetic analysis of FGFR1 signalling reveals distinct roles of MAPK and PLCgamma1 activation for self-renewal of adult neural stem cells. Mol Brain. 2009;2:16.

52. Kriventseva EV, Kuznetsov D, Tegenfeldt F, Manni M, Dias R, Simao FA, et al. OrthoDB v10: sampling the diversity of animal, plant, fungal, protist, bacterial and viral genomes for evolutionary and functional annotations of orthologs. Nucleic Acids Res. 2019;47(D1):D807-D11.

53. Lassmann T, Sonnhammer EL. Kalign--an accurate and fast multiple sequence alignment algorithm. BMC Bioinformatics. 2005;6:298.

54. Clayton DA, Shadel GS. Isolation of mitochondria from tissue culture cells. Cold Spring Harb Protoc. 2014;2014(10):pdb prot080002.

55. Valente AJ, Maddalena LA, Robb EL, Moradi F, Stuart JA. A simple ImageJ macro tool for analyzing mitochondrial network morphology in mammalian cell culture. Acta Histochem.

2017;119(3):315-26.

56. Martin-Maestro P, Gargini R, Garcia E, Perry G, Avila J, Garcia-Escudero V. Slower Dynamics and Aged Mitochondria in Sporadic Alzheimer's Disease. Oxid Med Cell Longev. 2017;2017:9302761. 
Figure 1
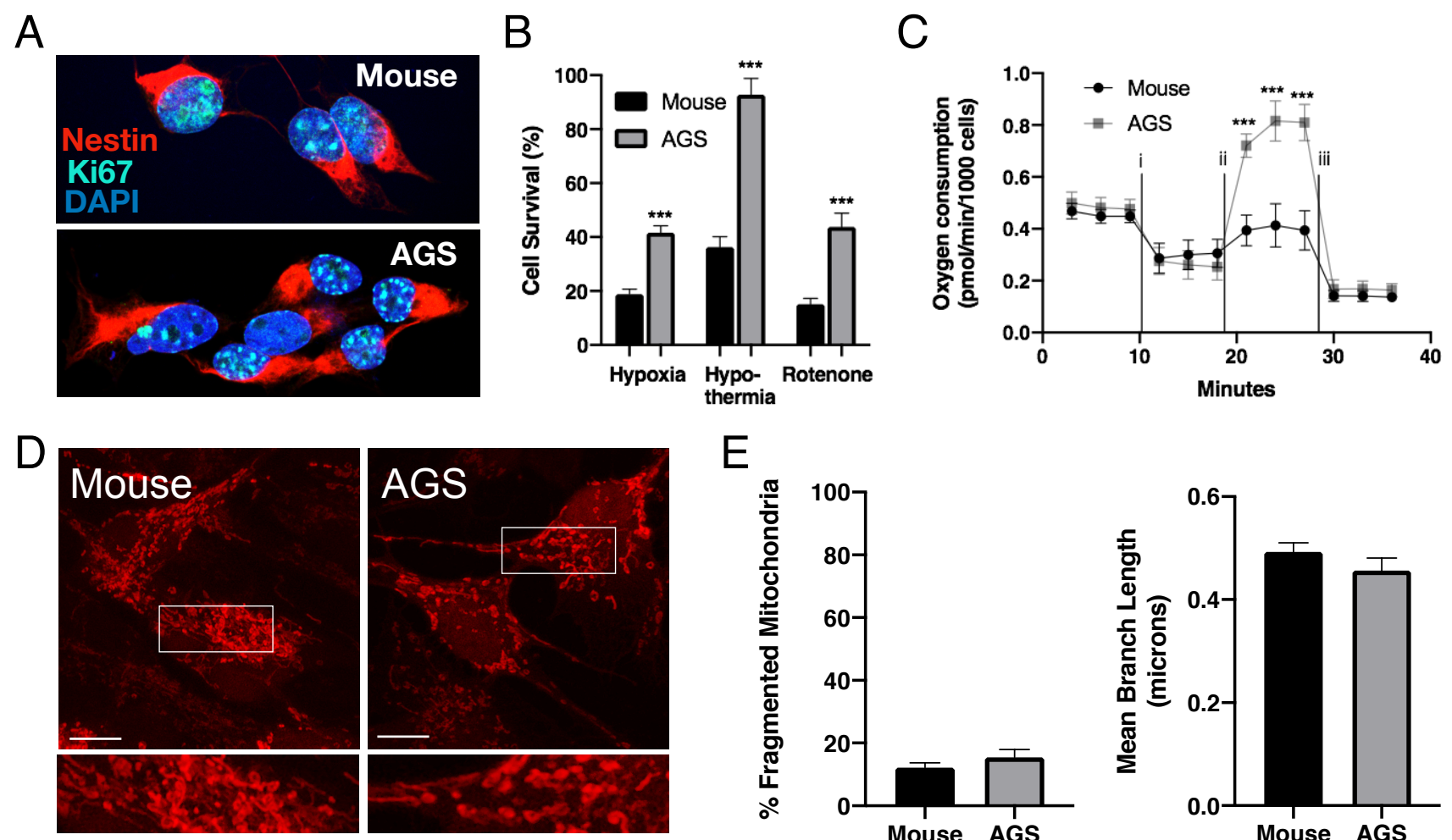

E
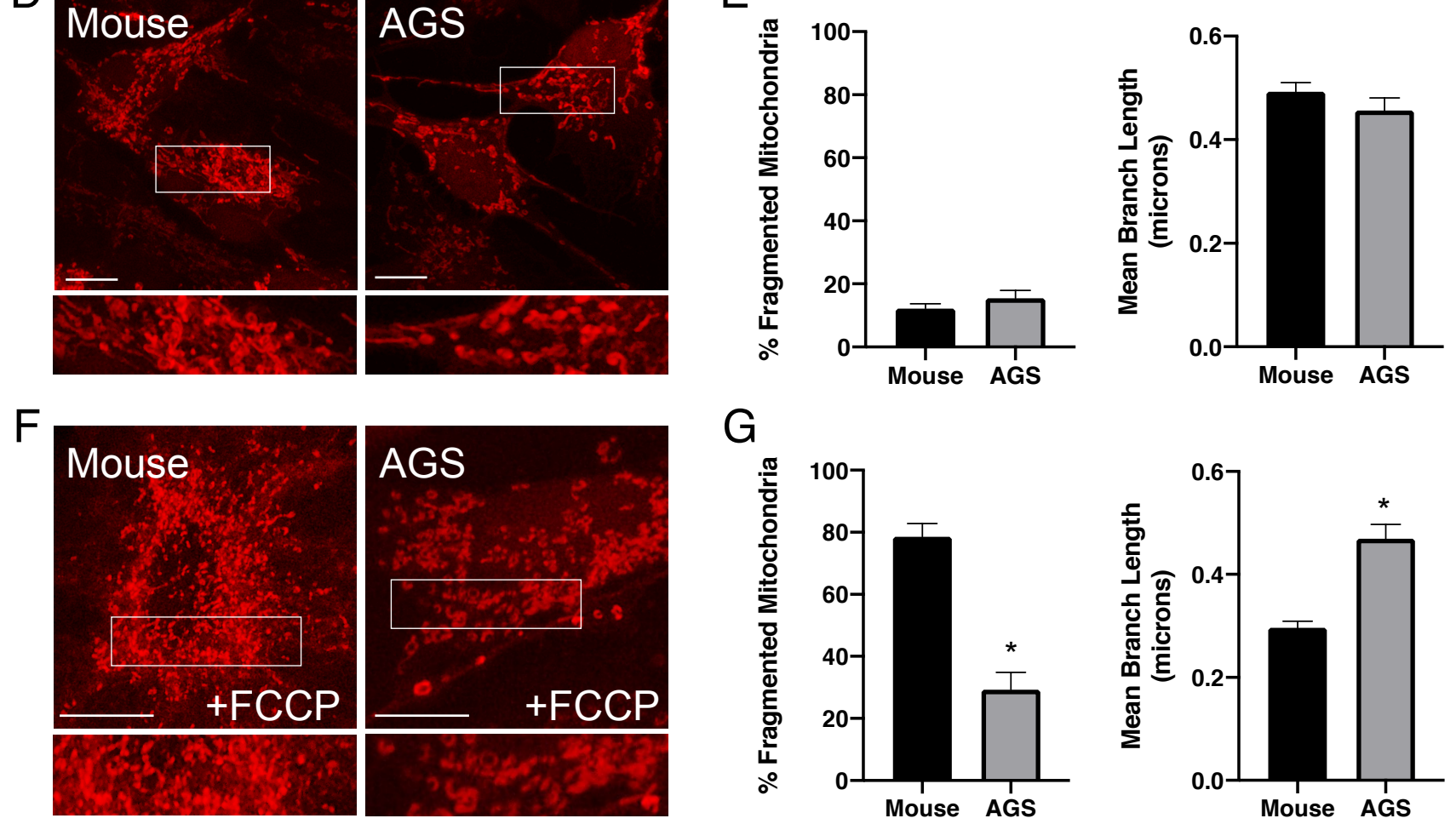

G
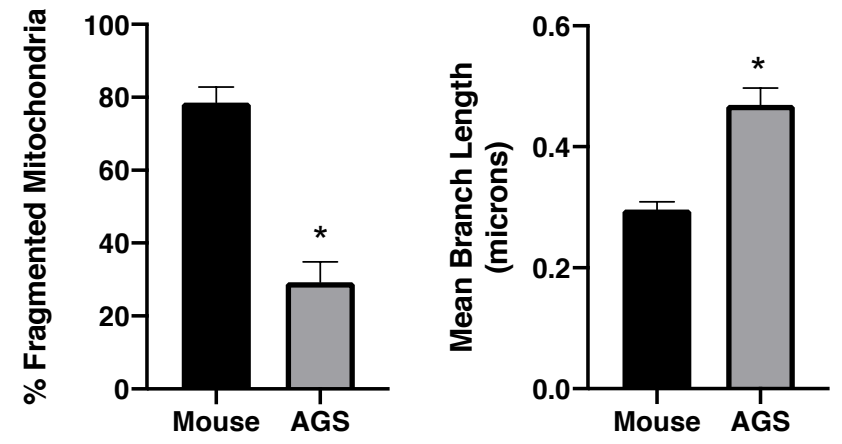


\section{Figure 2}

A

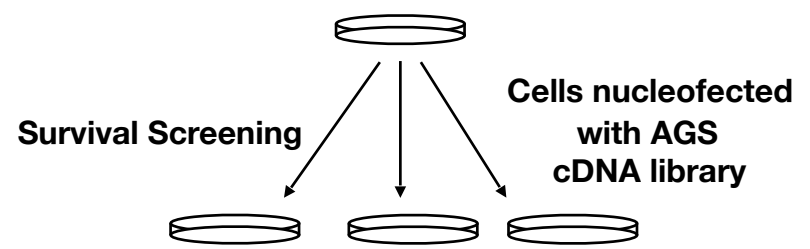

Hypoxia Hypothermia Rotenone

$(1 \%) \quad\left(31^{\circ} \mathrm{C}\right)$

D

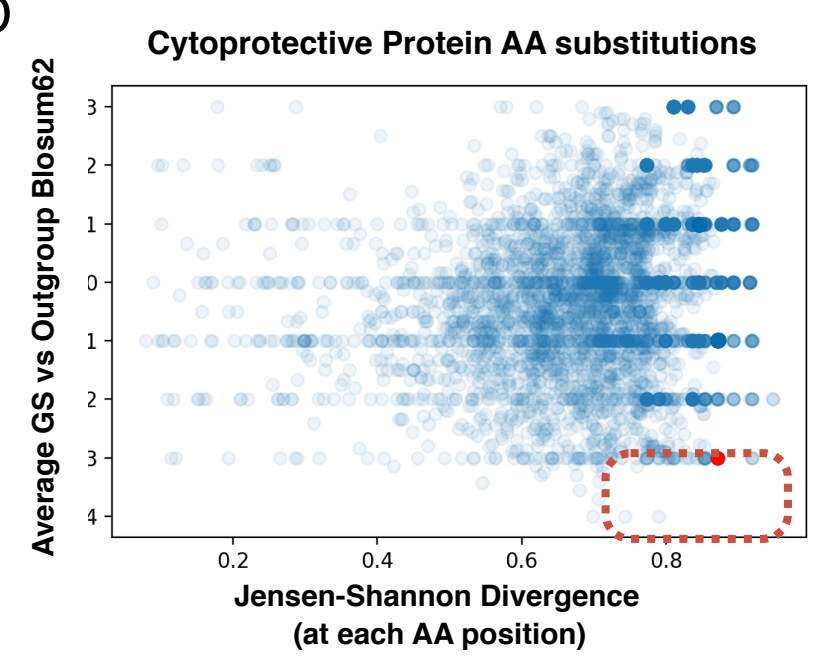

B

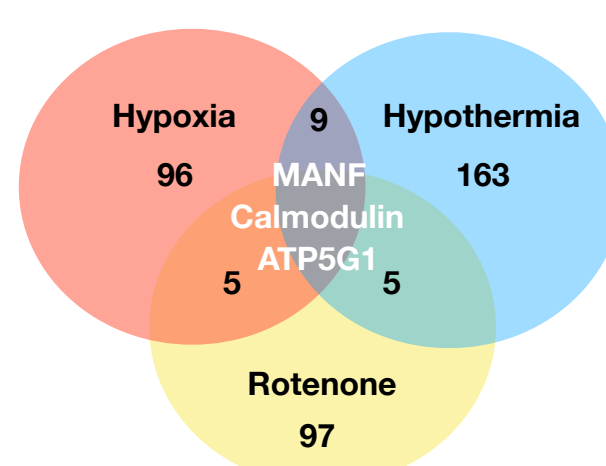

C

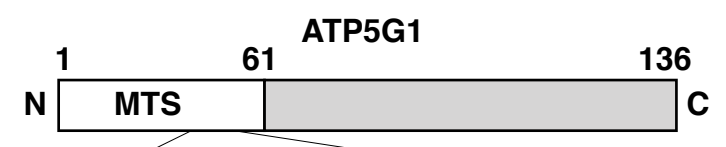

E

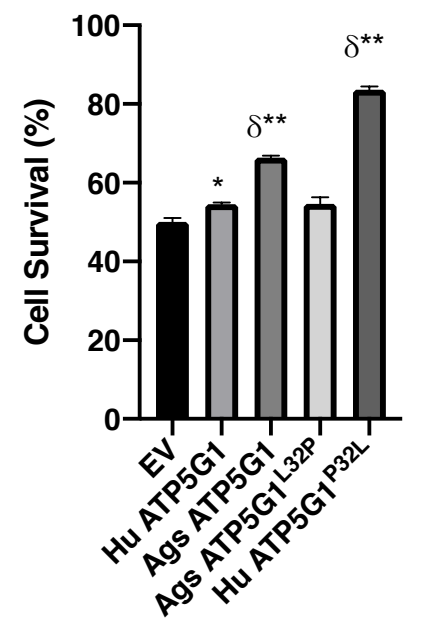

$\mathrm{F}$

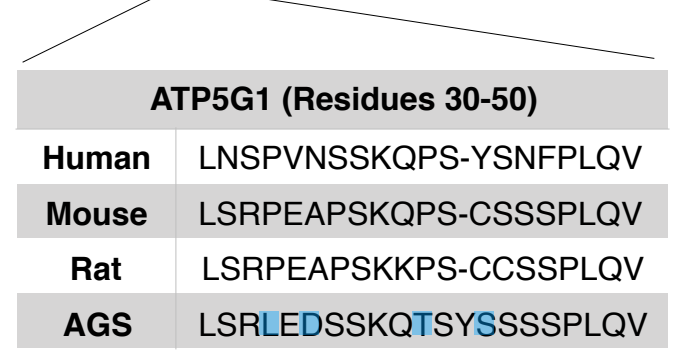

G

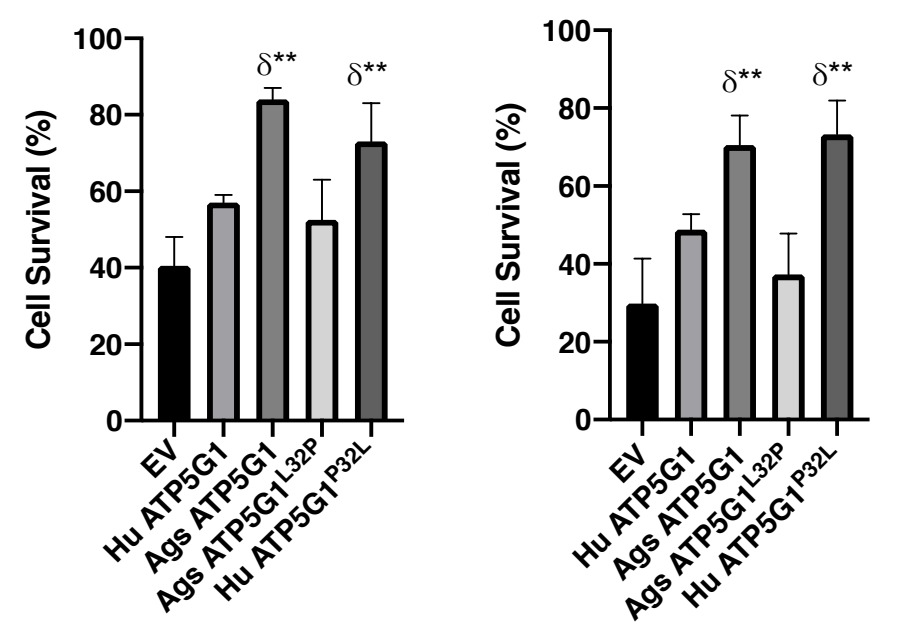


Figure 3

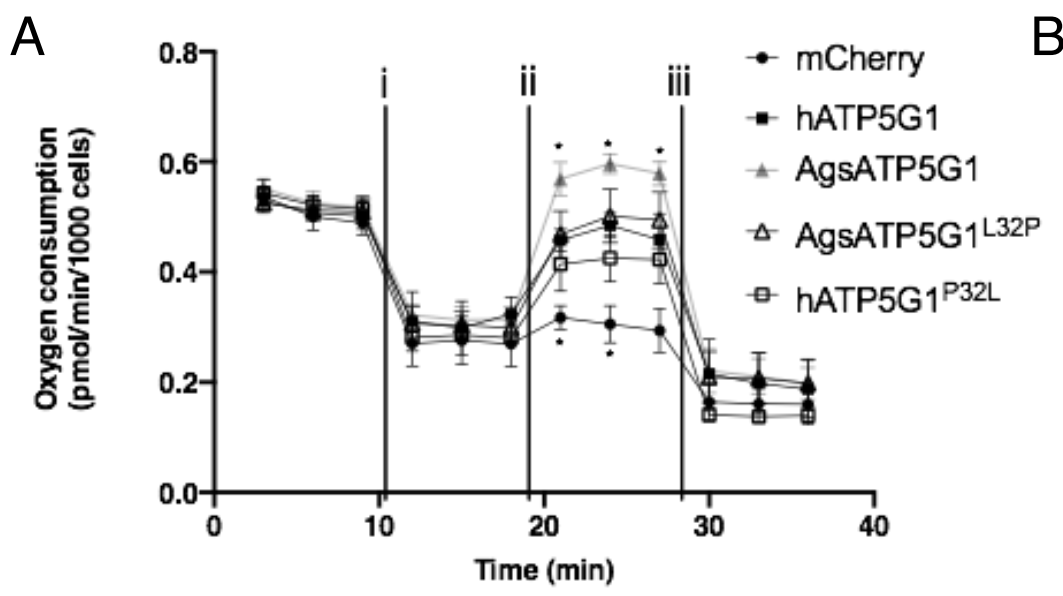

B
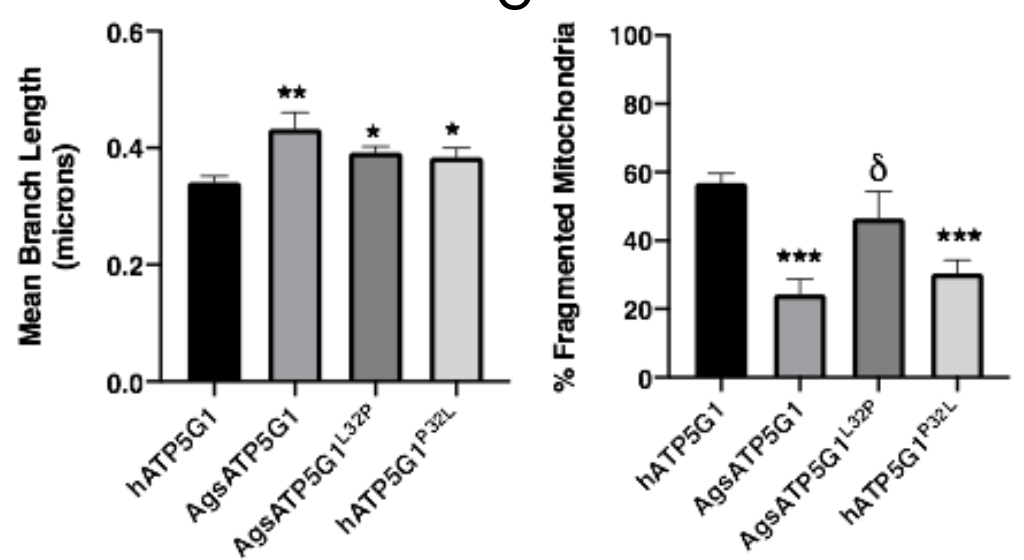

D
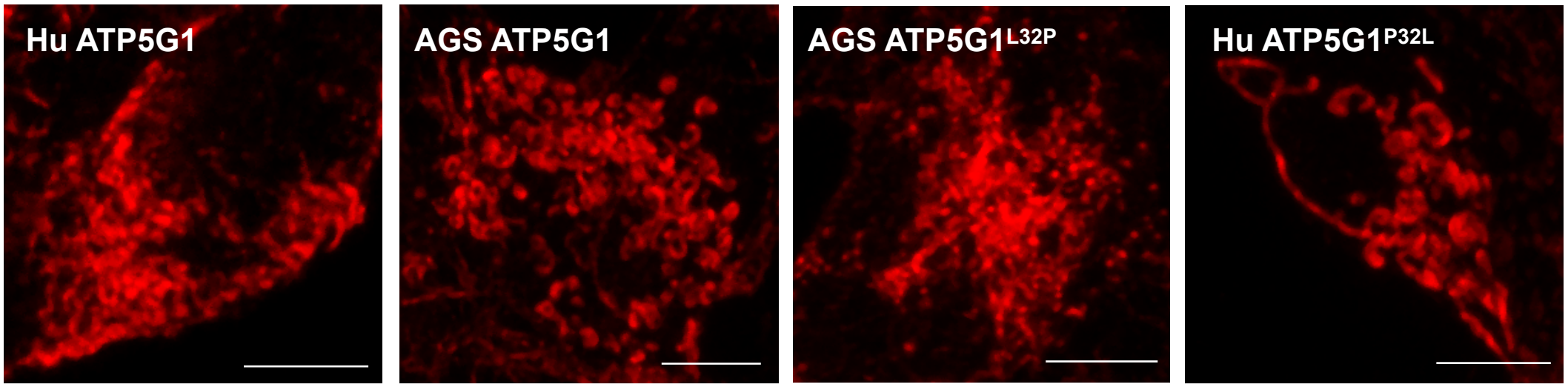
Figure 4

A

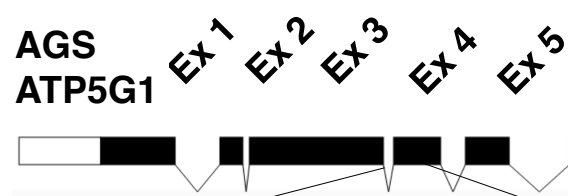

(+)Strand cctccttcCTGAATAGACTAGAGGACTC (-)Strand ggaggaagGACTTATCTGATCTCCTGAG

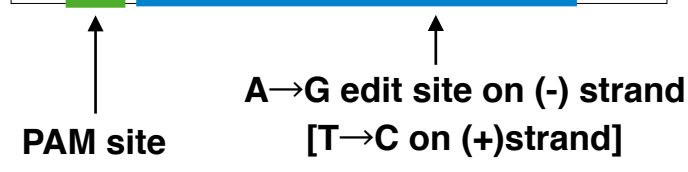

D

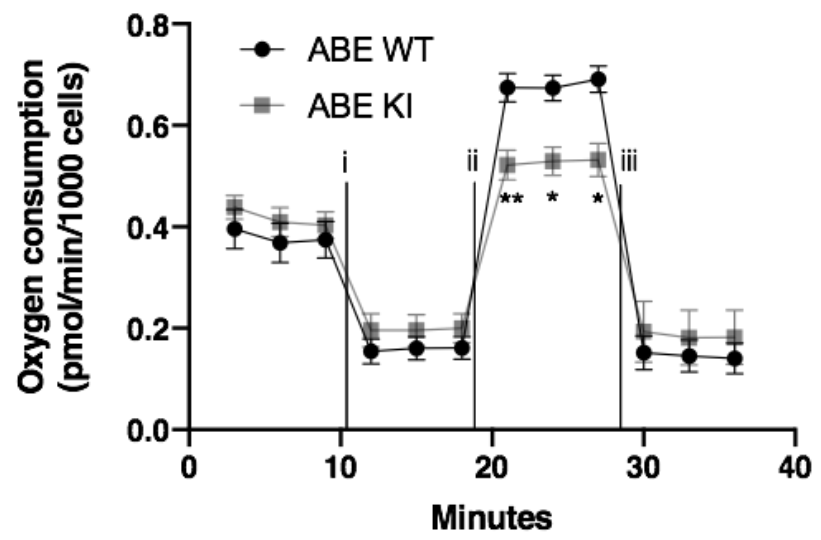

B

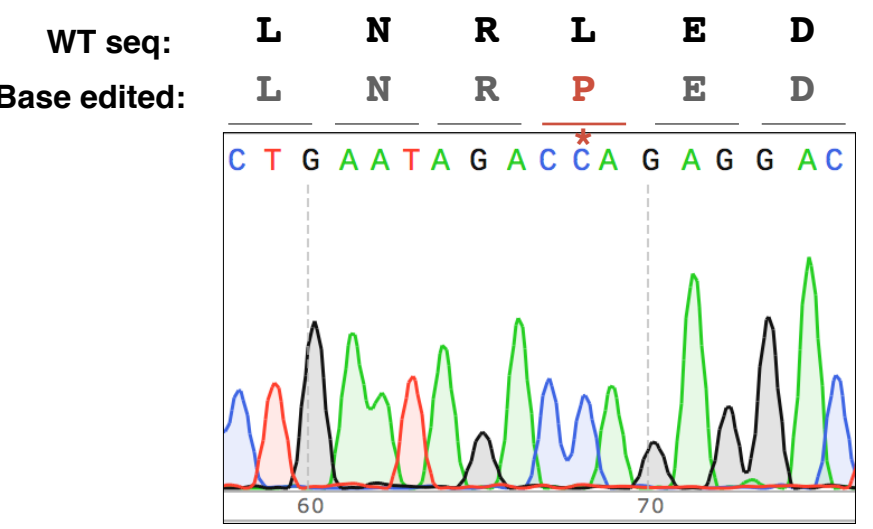

$\mathrm{F}$
C

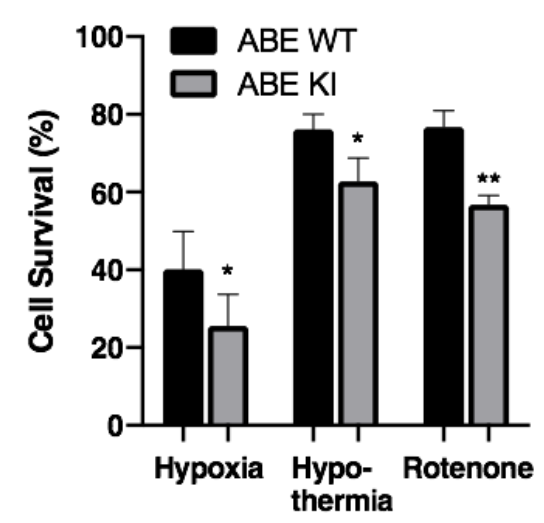

G
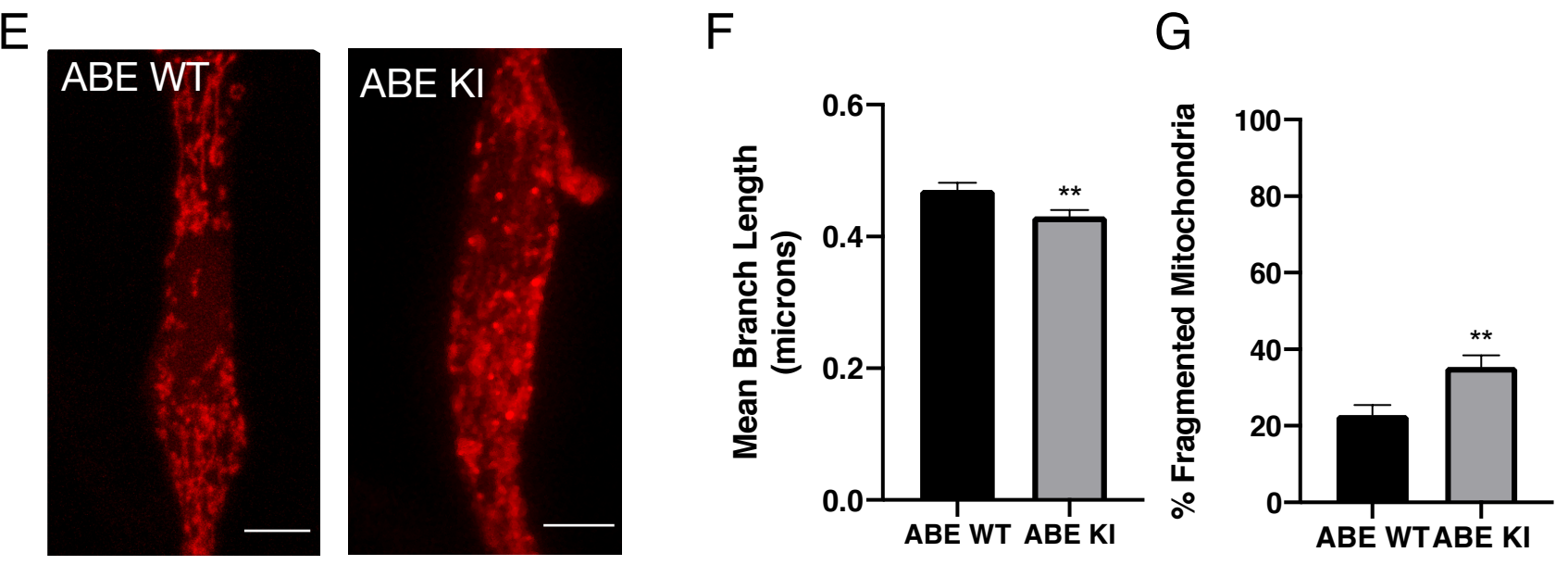
Figure 2 Supplement
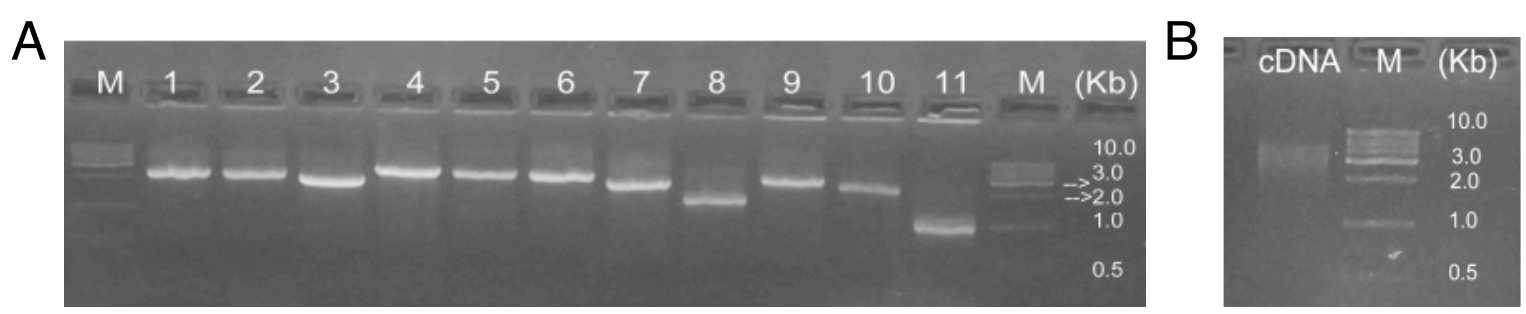

C ATP5G1 Sequence Alignment

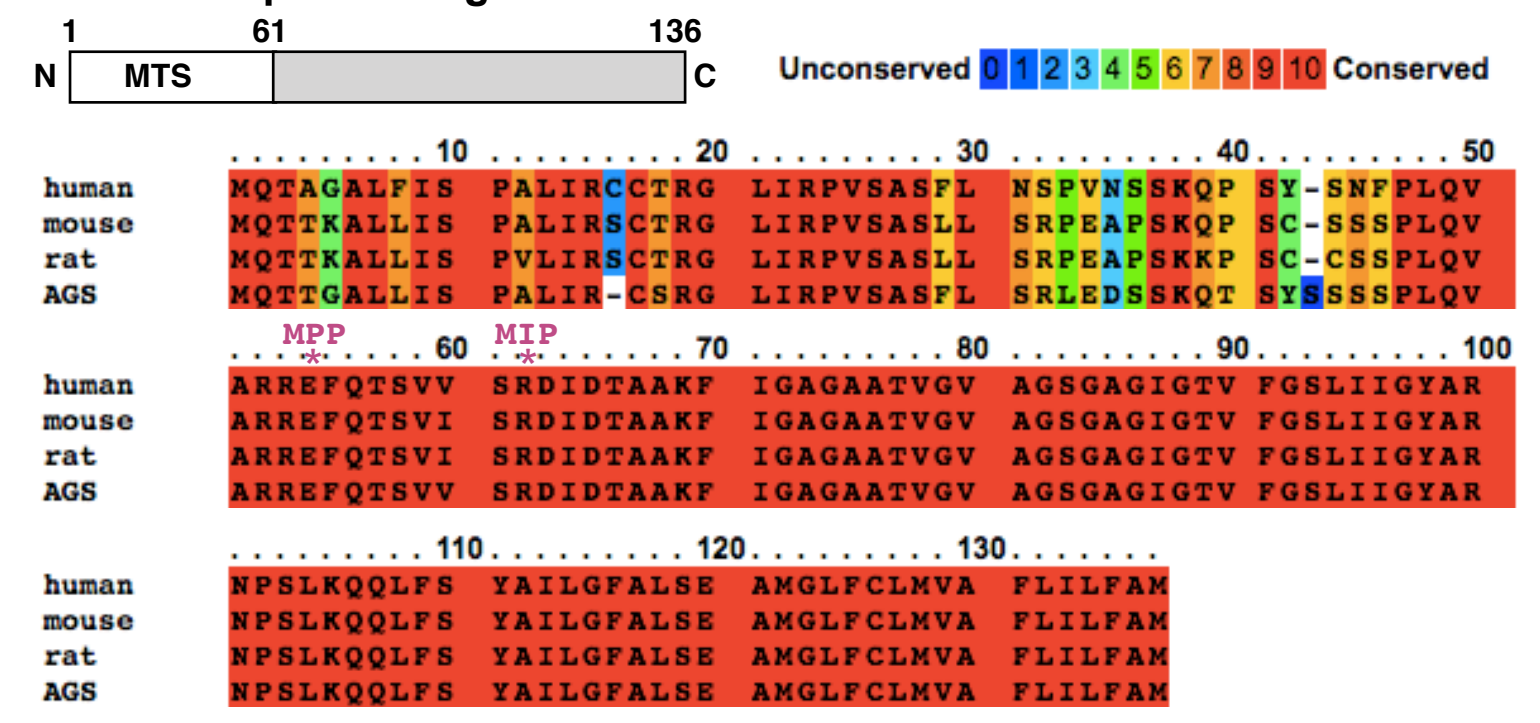

D

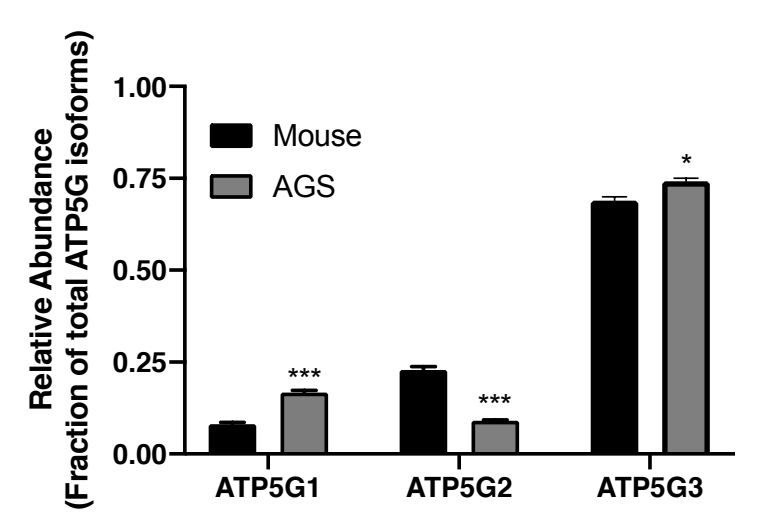

E

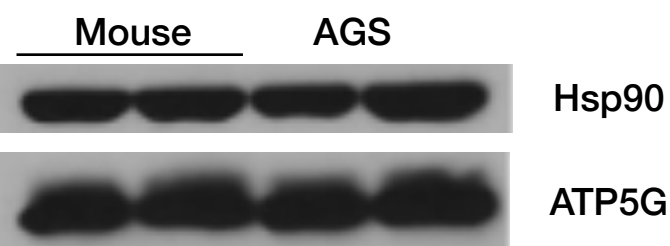

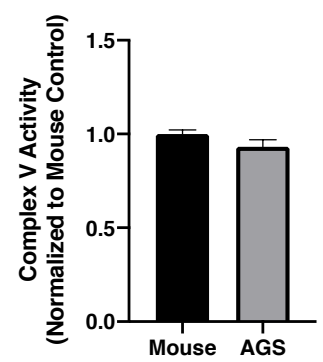


Figure 3 Supplement

A

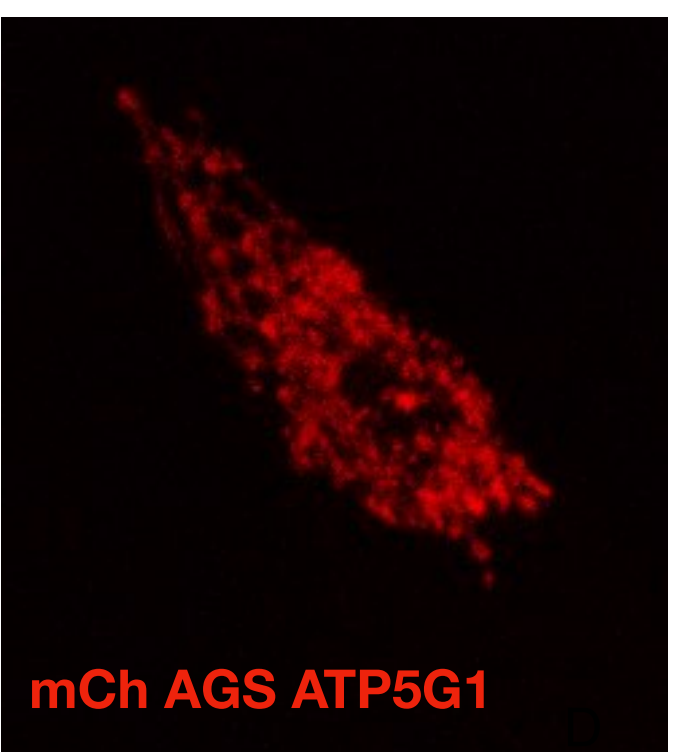

B

Hypoxia

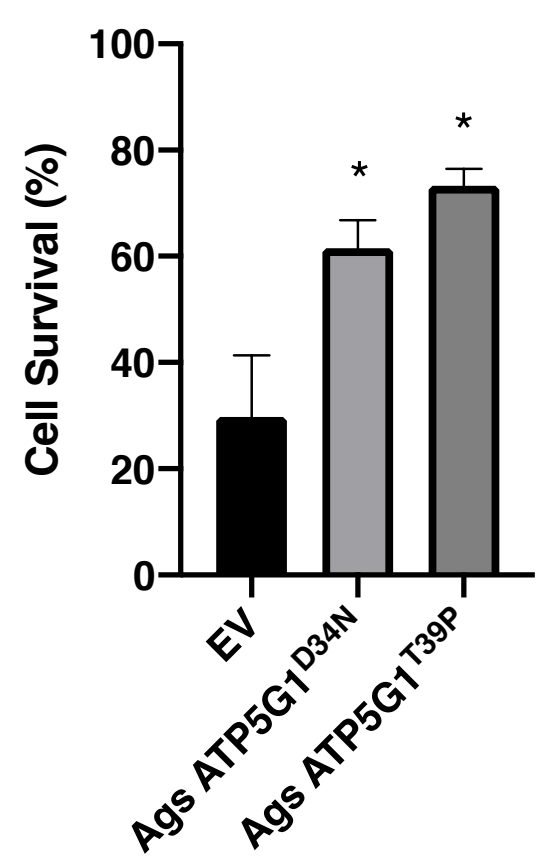

GFP Cox8

C

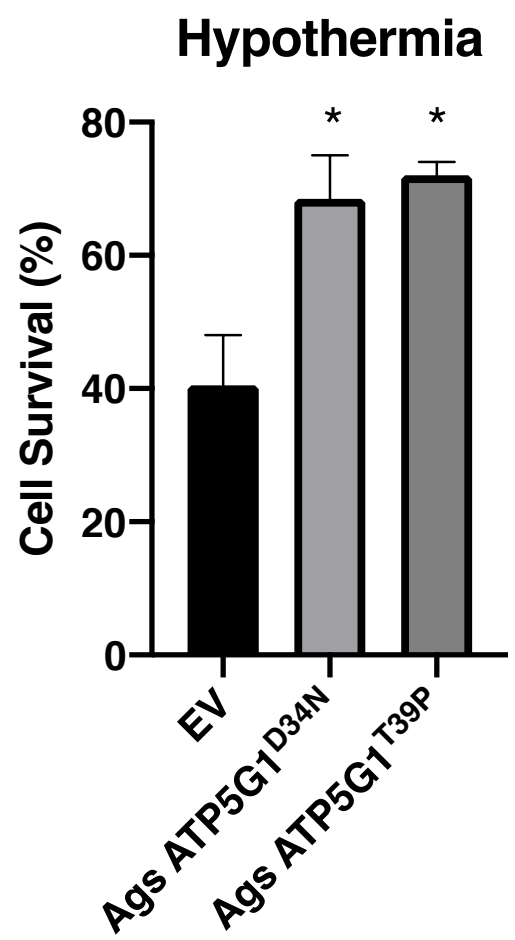

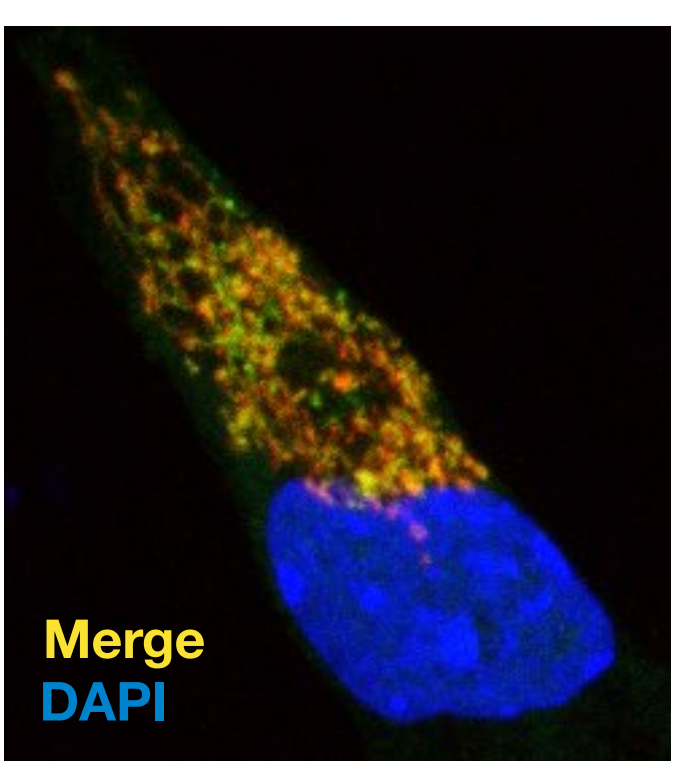

D

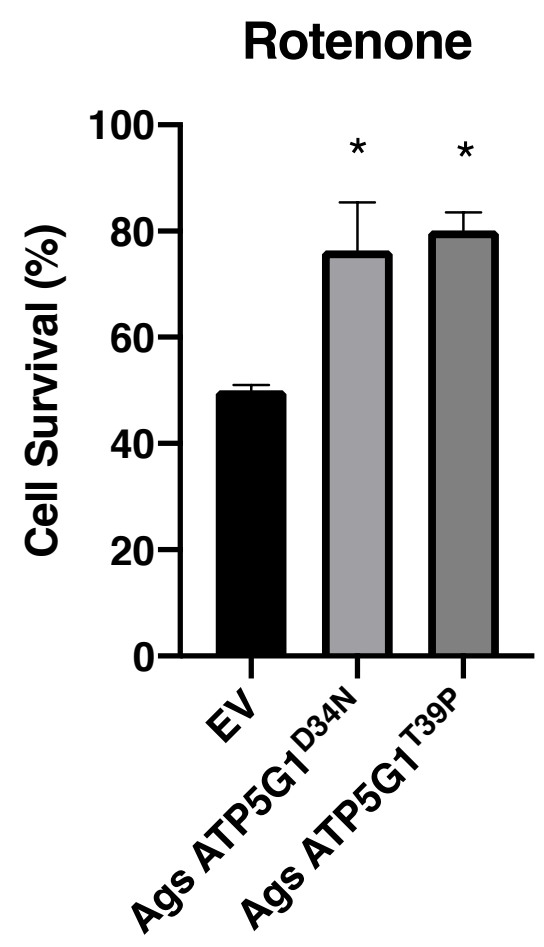


Figure 4 Supplement

A

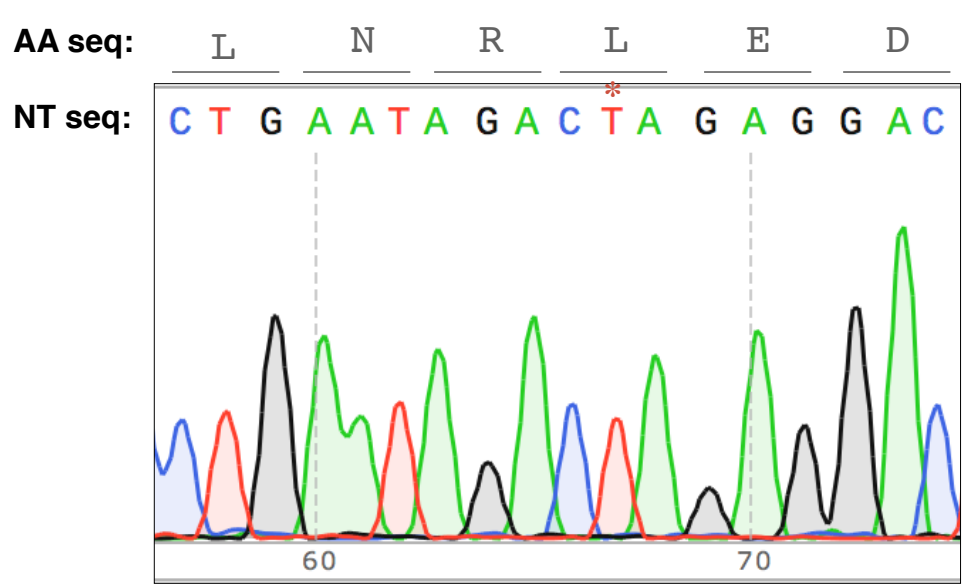

C

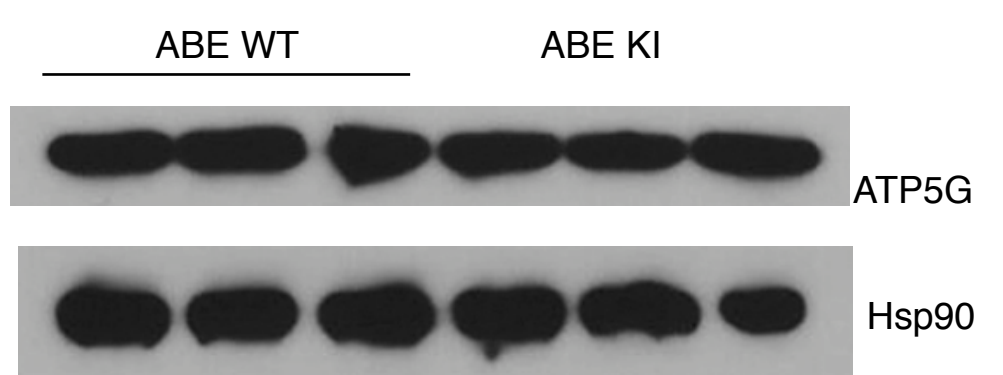

B

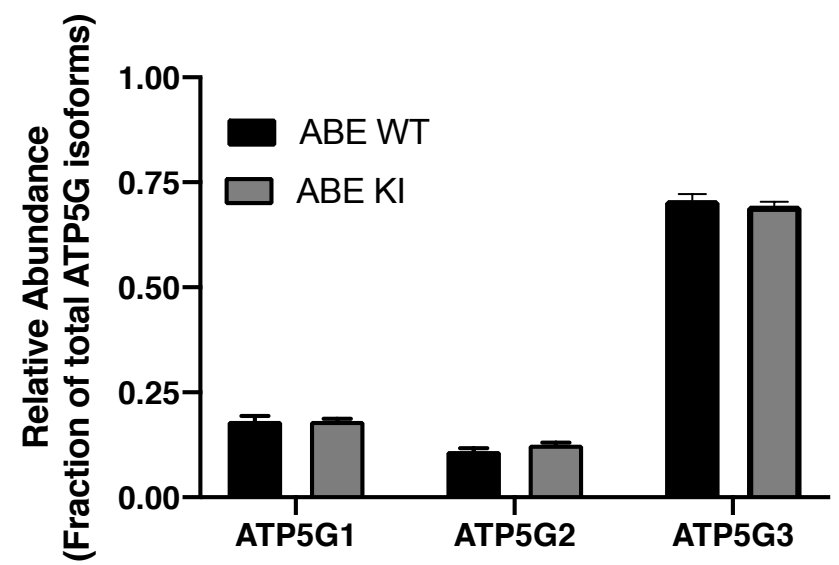

D

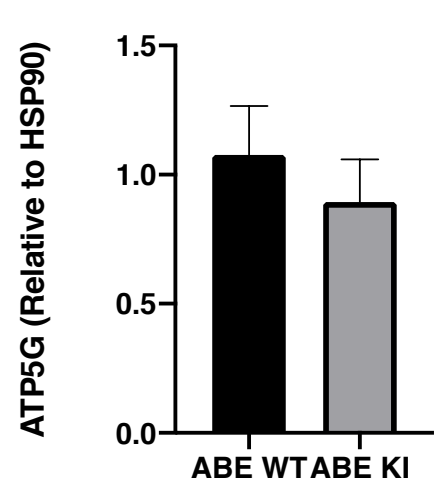

$E$

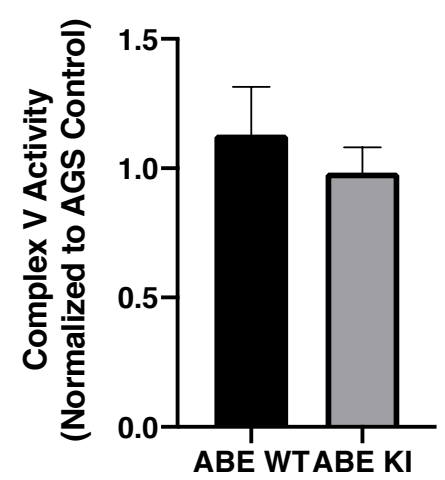


bioRxiv preprint doi: https://doi.org/10.1101/2020.01.31.929018; this version posted January 31, 2020. The copyright holder for this preprint (which was not certified by peer review) is the author/funder, who has granted bioRxiv a license to display the preprint in perpetuity. It is made available under aCC-BY-NC-ND 4.0 International license.

1 Supplemental Table 1. Species-specific primers used in quantitative RT-PCR.

2

\begin{tabular}{|l|l|}
\hline Mouse & \\
\hline Rpl27 Forward & ATA AGA ATG CGG CCG CAA GC \\
\hline Rpl27 Reverse & ATC GAT TCG CTC CTC AAA CTT \\
\hline Atp5g1 Forward & TGC AGA CCA CCA AGG CAC TG \\
\hline Atp5g1 Reverse & GGC CTC TGG TCT GCT CAG GA \\
\hline Atp5g2 Forward & CGT CTC TAC CCG CTC CCT GA \\
\hline Atp5g2 Reverse & CTG CAG ACA GCG GAC GAC TC \\
\hline Atp5g3 Forward & GGG CCC AGA ATG GTG TGT GT \\
\hline Atp5g3 Reverse & TGC AGC ACC TGC ACC AAT GA \\
\hline & \\
\hline AGS & \\
\hline Rpl27 Forward & CTG CCA TGG GCA AGA AGA AA \\
\hline Rpl27 Reverse & AGC AGG GTC TCT GAA GAC AT \\
\hline Atp5g1 Forward & TCC GGC TCT GAT CCG CTG TA \\
\hline Atp5g1 Reverse & GGG AGC TGC TGC TGT AGG AA \\
\hline Atp5g2 Forward & TGC CTG CTC CAG GTT CCT CT \\
\hline Atp5g2 Reverse & GGG ACT GCC AAG CTG CTG AA \\
\hline Atp5g3 Forward & TGA GGC CCA GAA TGG TGA ACG \\
\hline Atp5g3 Reverse & CAG CAC CAG AAC CAG CCA CT \\
\hline
\end{tabular}

